\title{
On the effect of the curing cycle on the creation of pores in structural adhesive joints by means of X-ray microtomography
}

\author{
V. Dumont (10) ${ }^{a, b}$, C. Badulescu ${ }^{a}$, G. Stamoulis ${ }^{c}$, J. Adrien $^{d}$, E. Maire ${ }^{d}$, A. Lefèvre ${ }^{b}$, \\ and $\mathrm{D}$. Thévenet ${ }^{\mathrm{a}}$ \\ aENSTA Bretagne, UMR CNRS 6027, IRDL, Brest, France; ${ }^{b}$ Safran Reosc - Engineering \& Integration \\ Department, Saint-Pierre-du-Perray, France; 'UMR CNRS 6027, IRDL, University Bretagne Occidentale, \\ Brest, France; dUMR CNRS 5510, Laboratoire MATEIS, University Lyon, INSA Lyon, Villeurbanne, France
}

\begin{abstract}
Adhesive bonding is increasingly used in numerous industrial branches (aeronautics, space, etc.) for the many advantages this technique features. The quality of the adhesive bonds depends on diversified factors, and bonding defects within the joints are common. These defects may take the shape of pores, of various sizes, which are a possible threat to the good mechanical strength of the joints. These pores may create unwanted stress concentra-tions, and they are susceptible to locally weaken the adhesive joints. As they are created during the bonding process, the hypothesis that the bonding and curing conditions should influ-ence their properties is wellfounded. In order to validate this assertion, adhesively bonded samples were made, for different adhesives and different curing cycles. In particular, the influence of the peak temperature reached during the curing cycle was studied. The pores in the joints were then observed and studied using X-ray microtomography. The performances of the segmen-tation technique developed for the data processing were studied beforehand on synthetic data, in order to identify the limitations of the suggested methodology and to quantify the uncertainty on the computed quantities. It was found that a higher curing temperature may induce noticeable pore growth phenomena (mainly dilation and coalescence). In particular, this growth seems to be predominant halfway-through the thickness of the joints, resulting in an observable decrease in the effective section.
\end{abstract}

\section{Introduction}

Structural bonding has known a surge in use and popularity in the past two decades, for several reasons: (i) this technique allows engineers to design lighter and more energy-saving structures, (ii) it is well-suited to multimaterials assemblies, (iii) it paved the road to mechanical assemblies using materials incompatible with traditional techniques (welding, bolting, riveting, etc.), and (iv) bonded structures feature fairly interesting stress distribution properties. Therefore, 
more and more industrial fields started to include this technique in their processes and in their designs, as in aeronautics, space, medical, etc. However, these advantages come with some drawbacks: the quality of the adhesive bond is sensitive to the bonding process, ${ }^{[1]}$ the mechanical behaviour of adhesives is highly non-linear, ${ }^{[2,3]}$ and the strength of the adhesive joint depends on a multitude of factors, related to both adhesion and cohesion phenomena. Due to the numerous elements impacting the quality of the joint, bonded structures are highly sensitive to defects which may occur during the bonding process. Among these commonplace defects that cannot be avoided in standard bonding conditions, one may reference the creation of pores throughout the mixing, bonding, and curing steps. These pores could possibly be a significant disturbance to the good mechanical resistance of the bond, as (i) they decrease the cross-section of the joint, (ii) the continuum and the stability of the adhesive are challenged and put at risk, (iii) they may induce unwanted stress concentrations, and (iv) they may lead to predisposed crack propagation paths within the material. As these voids within the material are created during the bonding and the curing of the adhesive, it is fair to hypothesise that the bonding conditions and the curing conditions should have an influence on their characteristics (mainly number, volume fraction, and size).

Unfortunately, as they are located inside the material, they are not easily detected and visualised. It is nonetheless possible to do so, using advanced imaging technologies such as X-ray microtomography. This technique has multiple advantages: (i) it is a non-destructive technology, (ii) it provides information from the bulk of opaque materials, (iii) the resolution can reach 1 $\mu \mathrm{m}$ (or even below) and (iv) the gathered data are three-dimensional. In the scope of materials science, it was at first used mainly on metallic alloys as in the work of Liu and Bathias ${ }^{[4]}$ on the influence of defects on both tensile and fatigue properties of an aluminium alloy reinforced composite. They showed that the presence of pores and misorientation of fibres had a significant influence on the decrease in terms of fatigue life, and therefore the authors bring to attention the importance of a carefully controlled manufacturing process regarding these aspects. Composite materials were also the subject of such measurements. It is also worth referencing the works of Hirano et al. who performed damage characterisation and assessment in 1995 using in-situ tomography measurements on aluminium alloy matrix composites with $\mathrm{SiC}$ fibre reinforcements. ${ }^{[5]}$

$\mathrm{X}$-ray microtomography is nowadays widely used in materials science papers, for the many advantages it features. These studies use tomography measurements mainly for two purposes: the characterisation of the processing conditions for given materials, ${ }^{[6]}$ and damage characterisation. Regarding this aspect, the possibility to perform in-situ X-ray tomography measurements, while a mechanical load is applied, is a definitive edge. In particular, the teams of Maire et al., and Adrien et al. took advantage of this tool to investigate the creation of damage in metals under tensile loading ${ }^{[7]}$ and in polymeric syntactic foams under compressive stress. ${ }^{[8]}$ In the 2007 study of Maire, ${ }^{[7]}$ it is notably 
shown that data from acoustic emission measurements and X-ray tomography measurements lead to similar conclusions in terms of crack and void detection, which validates the use of X-ray tomography for such purposes. It is also an interesting tool to build accurate finite element models, as it was done by Maire et al. ${ }^{[9]}$ in the case of metallic foams. It was then possible to reconstruct their structure down to the cellular level and to use this geometry in finite element softwares.

Regarding the study of polymers specifically, a few studies may be found in the literature. A topic often encountered is the fabrication of polymeric structures via additive manufacturing techniques, as they are prone to generate voids in the material. For instance, a 2016 paper by Pavan et al. characterised the porosity of laser-sintered polyamide structures, of various sizes. ${ }^{[10]}$ It was found that the size of the structure influenced the properties of the pores created during the process. More recently, Wang et al. proposed a micromechanical model in order to characterise the mechanical behaviour of 3D-printed polymers. ${ }^{[1]}$ Nonetheless, few studies may be found specifically on adhesives, and even more so regarding adhesively bonded assemblies. This is probably explained by the a priori low risk of pores creation for these materials when compared to additive manufactured polymers.

As far as the field of structural bonding is concerned, X-ray tomography has been used on bonded wooden structures in order to characterise the bonding process and the adhesive penetration in the wood by McKinley et al. ${ }^{[12]}$ Still in the context of wooden structures, Schwarzkopf ${ }^{[13]}$ coupled tomography observations with numerical simulations to describe the micromechanical strains in the joints and in the substrates, where the adhesive tends to diffuse due to the fibrous nature of wood. This results in a fairly accurate description of the kinematics of the interfaces, even though the authors highlight a few limitations, such as the commonplace reconstruction artefacts. No particular attention has been given so far to the microstructural characterisation of adhesive joints through this technique, and the use of $\mathrm{X}$-ray microtomography is hence fairly new in the field of structural bonding for this type of subject, as opposed to other materials: minerals, ${ }^{[14]}$ food, ${ }^{[15]}$ composites, ${ }^{[16,17]}$ etc. Nonetheless, a 2019 paper by Dumont et al. investigated the evolution of the microstructural characteristics of adhesive joints under mechanical stress by means of in-situ X-ray microtomography.

In the following study, the authors investigate the effects the curing process may have on the pores within adhesive joints. This study is performed using two different bicomponent epoxy adhesives, for two curing cycles, with means of $\mathrm{X}$-ray microtomography. First, the samples and their preparation are detailed. Then, the experimental procedure for X-ray microtomography measurements is presented. The authors also describe the tomographic data segmentation tool which was developed and discuss its performances and abilities using synthetic 
data, before its use on experimental data. Lastly, the experimental results are outlined, along with a discussion on the observed phenomena.

\section{Motivation}

The observation of failure profiles of ruined samples after tensile tests through an optical microscope (Figure 1a) and a scanning electron microscope (Figure 1(b,d)) highlights the presence of pores in the material. Moreover, these pores seem to play a part in the failure of the samples, as it is illustrated in detail by Figure 1(a,c and d) where cracks can be spotted in the neighbourhood of pores.

Even if any characterisation is impossible this way due to the lack of provided information, it is fairly easy to visualise the creation and the growth of pores within an adhesive using a mere optical microscope. In a first stage, a layer of bicomponent epoxy adhesive (Araldite $420 \mathrm{~A} / \mathrm{B}$ from the German manufacturer Huntsman ${ }^{T M}$ ) is spread between two sets of glass sheets, to be

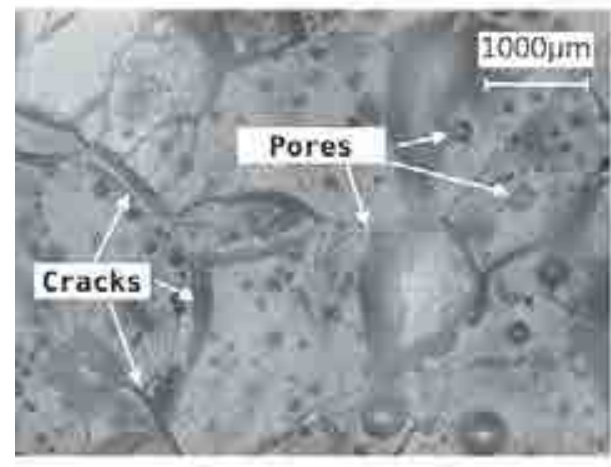

(a) Optical microscope view

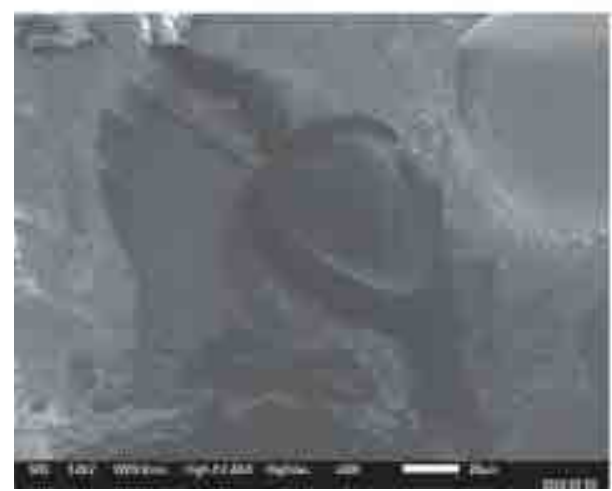

(c) Cracked pore (SEM - secondary electrons)

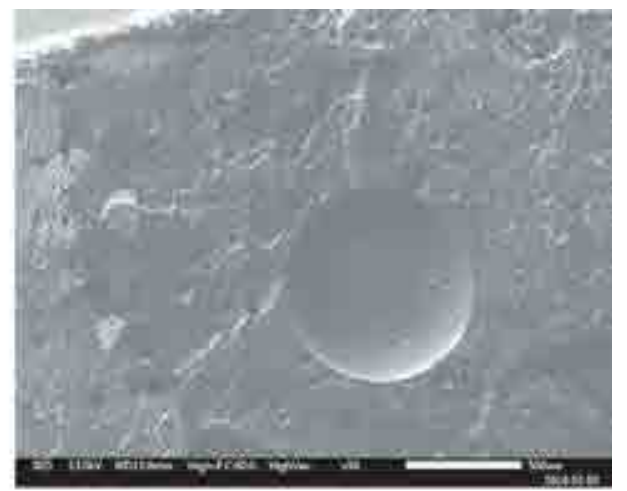

(b) Scanning Electron Microscopy (SEM - secondary electrons) view

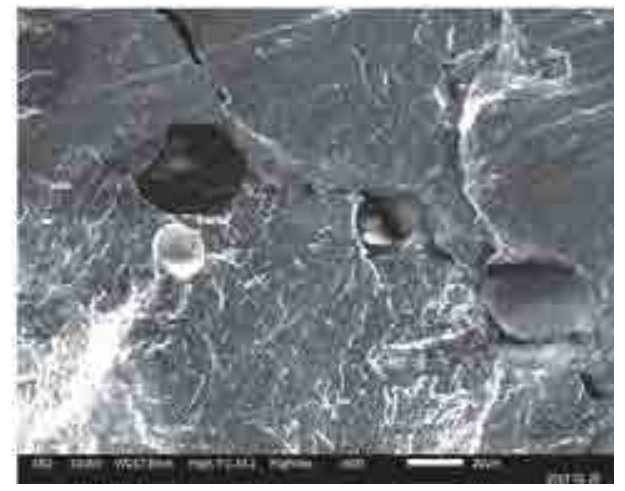

(d) Crack propagation through pores (SEM - secondary electrons)

Figure 1. Microscopic observations of failure profiles of adhesively bonded samples (adhesive B tensile tests). 
observed using a Keyence ${ }^{T M} V H X$ electronic optical microscope. The components of this adhesive were mixed together using a planetary mixer, so as to obtain a homogeneous material. One of these glass-adhesive-glass assembly was then left at ambient temperature for 12 hours, and the other one was polymerised at high temperature for a short period of time $\left(1 \mathrm{~h} 10\right.$ at $\left.110^{\circ} \mathrm{C}\right)$. They were then put back under the microscope. The picture of these samples can be found in Figure 2.

It is then quite obvious that the high curing temperature used for the material in Figure 2(a,c) had a significant influence on the pores inside the adhesive: they greatly increased in volume, and decreased in number, meaning that coalescence phenomena were very likely to happen during the polymerisation. Also, it is possible to detect these pores even in the initial state (Figure 2a), meaning that they are created as soon as the joint is formed (and possibly as soon as the components of the adhesive are mixed).

Moreover, it is possible to detect lighter, circular areas on these pictures: these denote the presence of glass beads in the adhesive, as it is common for

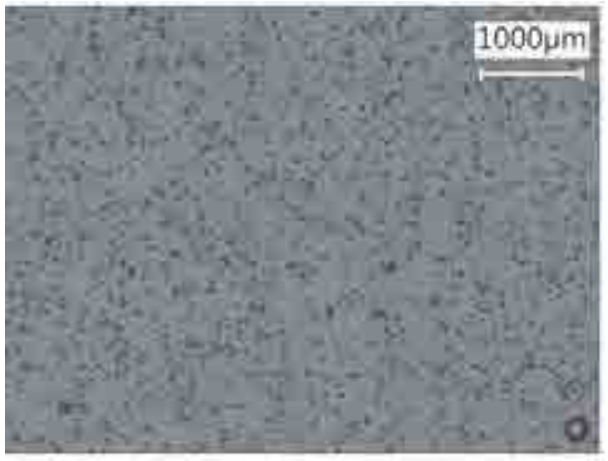

(a) Pores created within an adhesive joint before curing

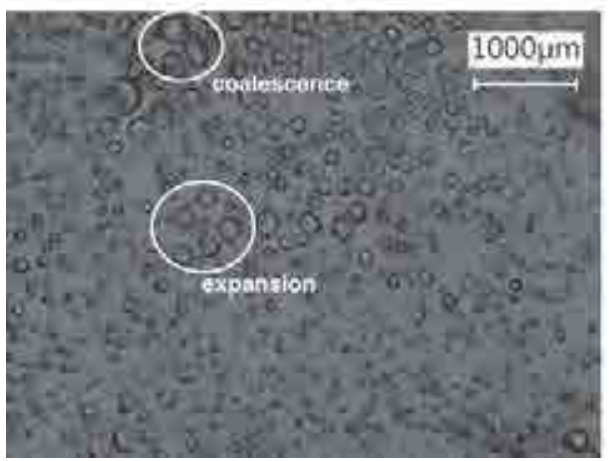

(c) Pores within an adhesive joint after $1 \mathrm{~h} 10$ at $110^{\circ} \mathrm{C}$

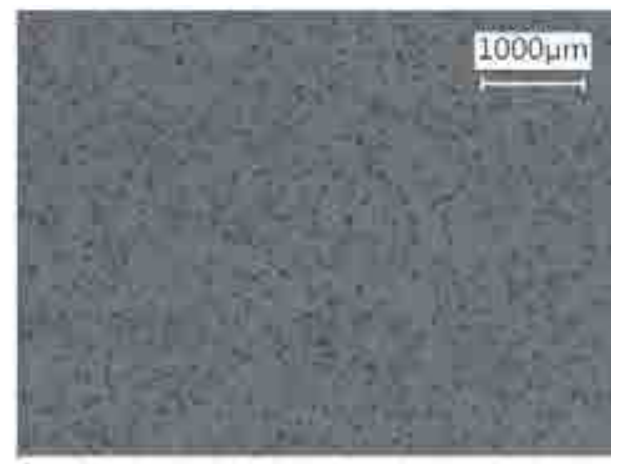

(b) Pores within an adhesive joint after $12 \mathrm{~h}$ at ambient temperature

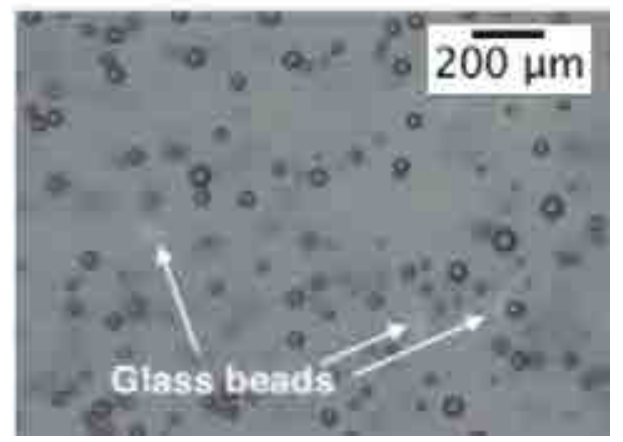

(d) Glass beads spotted on microscopy pictures (extracted from Fig.2a)

Figure 2. Highlighting the presence of pores in the adhesive as soon as the joint is shaped using an optical microscope. 
various reasons (mainly, to guarantee a minimal thickness for adhesive joints). Such beads are shown in Figure 2d, as an example. The adhesive used here is also used in the following microtomography measurements and is referred to adhesive $B$.

As stated in preamble, these microscopy techniques only allow for visualisation, and they are not suitable for characterisation. X-ray tomography is a fairly interesting answer to this defect, as it features high-resolution data acquisition, three-dimensional visualisation and measurements-oriented capabilities. These pores being structural defects, the assumption that they have an influence on the mechanical properties of the material seems reasonable, as expressed in $^{[18]}$ for composite materials. The conditions about this influence (in terms of pores size, number, volumetric ratio, etc.) are however not investigated in this study.

\section{Preparation of the specimen}

\subsection{Samples geometry}

The geometry of the samples must meet two criteria, to remain in the scope of this study: (i) they must be bonded assemblies (as opposed to bulk samples), and (ii) their dimensions must be adequate to the resolution of the X-ray microtomography measurements to be performed. Bonded samples are required as this study on the effect of curing is part of a more complete and extensive tests campaign performed on bonded assemblies. In order to check these requirements, miniScarf samples (Figure 3c) will be used. These samples are made out of traditional aluminium 2017A Scarf samples, where a rake-like shape is machined using waterjet cutting (Figure 3a). They are to be bonded using two different bicomponent epoxy adhesives, whose general characteristics are presented in Table 1). The adhesives are spread on each bonding surface of this pattern, and the Scarf samples (Figure 3a) are then assembled for the curing (Figure 3b). After the curing of the adhesive, each individual is then water-jet cut from the main structure to form the mini-Scarf samples, whose dimensions are presented in Figure 3c.

Moreover, in order to install the samples in a device for subsequent X-ray tomography in-situ tensile tests, threaded holes are accommodated at each end of the samples (Figure 3c). In the following study, no mechanical loading will be applied, but the aforementioned tensile machine is used as a mounting setup.

\subsection{Bonding and curing}

The substrates are prepared so as to remove any potential impurities, oily components, dirt, and oxides: (i) they are first degreased with acetone, (ii) a mechanical treatment (grade 180 sandpaper) is then applied, and (iii) another acetone cleaning is performed. 


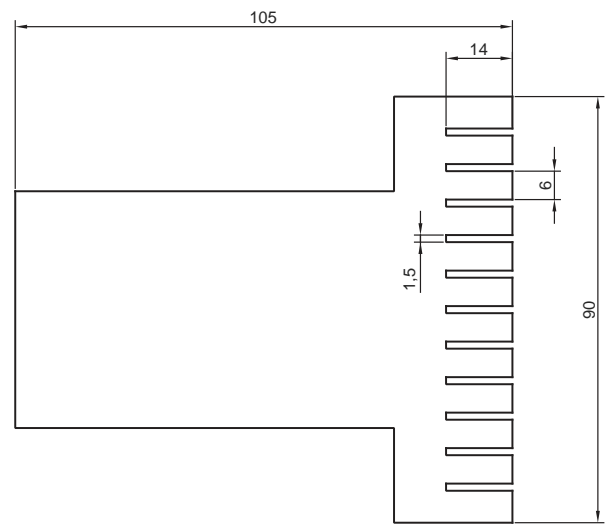

(a) Mini-Scarf samples layout (dimensions in $\mathrm{mm}$ )

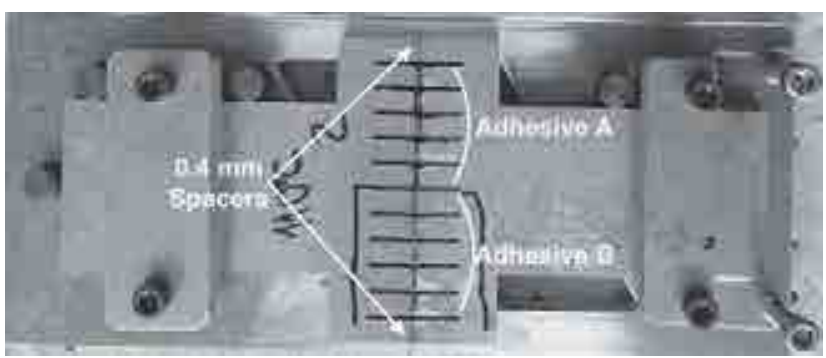

(b) Bonded modified SCARF samples

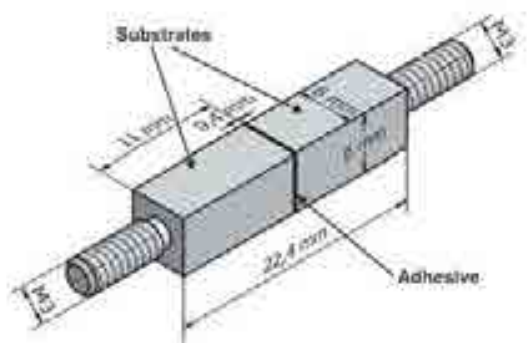

(c) In-situ microtomography tensile samples geometry

Figure 3. Modification of SCARF geometries for in-situ tomography applications.

As it has been evoked in introduction, two different adhesives will be tested in this study; hereafter they will be referred to as adhesive A and adhesive B (namely, Huntsman Araldite $420 \mathrm{~A} / \mathrm{B}$ ). The commercial reference of the adhesive A will not be expressed in this paper for confidentiality reasons. Both are bicomponent epoxy adhesives. Some general properties about these materials may be found in Table 1, as provided by the suppliers. The glass transition temperatures in Table 1 were measured using Differential Scanning Calorimetry (DSC). 
Table 1. A few properties for the considered adhesives.

\begin{tabular}{lcc}
\hline Property & Adhesive A & Adhesive B \\
\hline Texture & paste-like & honey-like \\
Known fillers & - & Glass beads \\
Glass transition temperature (DSC) & $\sim 90^{\circ} \mathrm{C}$ & $\sim 60^{\circ} \mathrm{C}$ \\
Young's modulus [MPa] & 1400 & 2000 \\
Poisson ratio [-] & 0.33 & 0.41 \\
Coefficient of Thermal Expansion $\left[\mathrm{ppm} .{ }^{\circ} \mathrm{C}^{-1}\right]$ & 83 & 78 \\
\hline
\end{tabular}

The components of the considered adhesives are mixed according to the guidelines provided by the manufacturers. The mixing is performed using a planetary mixing device, at $1500 \mathrm{rpm}$ for 7 minutes. These parameters are arbitrary, but chosen for the resulting product to be homogeneous (i.e. the hardener and the resin are visually indissociable).

In spite of the variation in terms of texture (Table 1), the application on the substrates follows the same pattern no matter the adhesive: a layer of adhesive is spread using a stainless steel spatula on both the substrates constituting a sample, which are then assembled (Figure $3 \mathrm{~b}$ ). The excess of adhesive dripping from the joint is cautiously cleaned. The alignment of the substrates and the thickness of the joints (set to $0.4 \mathrm{~mm}$ ) are controlled using a specially designed setup (Figure $3 b$ ).

Two curing cycles are performed, in order to investigate the effects of this factor on the resulting pores in the adhesives. Their characteristics may be found in Table 2.

The curing is done with a Memmert UF110+ ${ }^{T M}$ thermal chamber. It should be noted that these cycles yield a satisfyingly polymerised material for both adhesives, even though they are different in durations and temperatures. This was checked with DSC measurements, by comparing the crosslinking enthalpies of the uncured and cured material. It should be noted that, even though it is advised to cure such adhesives above their maximal glass transition temperatures, ${ }^{[19]}$ the low-temperature cycle has been designed to meet other additional requirements whose details are out of the scope of this study. Nonetheless, even if the high temperature configuration features a slightly higher curing degree, the low temperature curing configuration displays curing degree higher than $95 \%$, leading to the assumption that both cycles yield rather similar materials.

Once the curing cycle completed, the samples were water-jet cut from their Scarf base, to create microtomography individual samples such as presented in Figure $3 \mathrm{c}$. For the present study, one sample is considered per case. It should be

Table 2. Curing cycles steps and temperatures.

\begin{tabular}{lcc}
\hline Cycle & Step 1 & Step 2 \\
\hline Low temperature & $23^{\circ} \mathrm{C}$ for $24 \mathrm{~h}$ & $80^{\circ} \mathrm{C}$ for $2 \mathrm{~h}$ \\
High temperature & $110^{\circ} \mathrm{C}$ for $1 \mathrm{~h} 10$ & - \\
\hline
\end{tabular}


noted that two other specimens were examined for the following configurations: adhesive A cured at low temperature, and adhesive B cured at high temperature. A very low scattering was found in terms of porosity and number of pores. Consequently, it was decided to keep the number of investigated samples to one for each test case, in order to reduce the CPU usage on the computing cluster used to process the tomographic results.

\section{Experimental procedure}

\subsection{X-ray tomography principle}

Microtomography is an imaging technique which offers the possibility to reconstruct the internal structure of an object without causing any damage. This non-destructive characteristic is a significant advantage in materials science, in the case of in-situ acquisition for instance, as extensively presented by Buffière. ${ }^{[20]}$ Moreover, it is superior to many other imaging techniques such as any microscope-based methods, as it provides three-dimensional data, with a resolution down to $1 \mu \mathrm{m},{ }^{[20]}$ and even below, depending on the X-ray source used in the tomograph.

Tomography is based upon the variation of the linear X-ray attenuation coefficient $(\mu)$ within a material. For anisotropic materials, such as composites or foams, $\mu$ depends on the spatial coordinates $(x, y, z)$. Hence, building the threedimensional map of $\mu(x, y, z)$ is equivalent to reconstruct the internal microstructure of the observed sample. More detailed information may be found concerning the theoretical background of tomography imaging. ${ }^{[21]}$ The sample to be studied is mounted on a rotary platform (Figure 4), which incrementally performs a $360^{\circ}$ rotational movement in order to perform the data acquisition on a variety of organised propagation paths for the X-ray beams. The reconstruction of the volume is then performed using a Fourier transform-based algorithm. Extensive details on the theory of the reconstruction techniques may be found in the reference work of Kak. ${ }^{[21]}$

\subsection{Test procedure}

The samples are placed in the chamber of a Phoenix VtomeX laboratory tomograph, which contains a Varian Paxscan X-ray detector featuring a resolution of $1920 \times 1536$ pixels. This detector outputs a 14-bits coded greyscale pictures (or radiograms, see Figure 4) of the attenuation. The tensile machine in the chamber of the tomograph (Figure 5) is used as a mounting setup for the samples.

The voxel size obtained using this experimental set-up is $4.5 \mu \mathrm{m} \times 4.5 \mu \mathrm{m} \times 4.5$ $\mu \mathrm{m}$, which suits the typical size of the pores that are expected. Moreover, the glass beads included in the adhesive B feature a diameter above $60 \mu \mathrm{m}$ according to SEM observations, meaning that they will not be difficult to detect. 


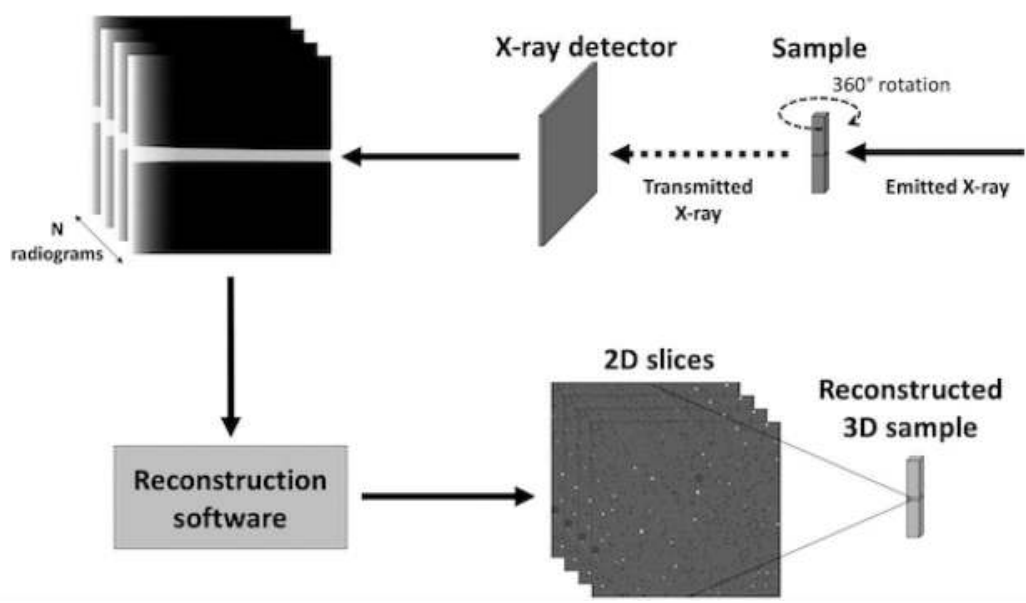

Figure 4. Tomography measurements principle. ${ }^{[22]}$

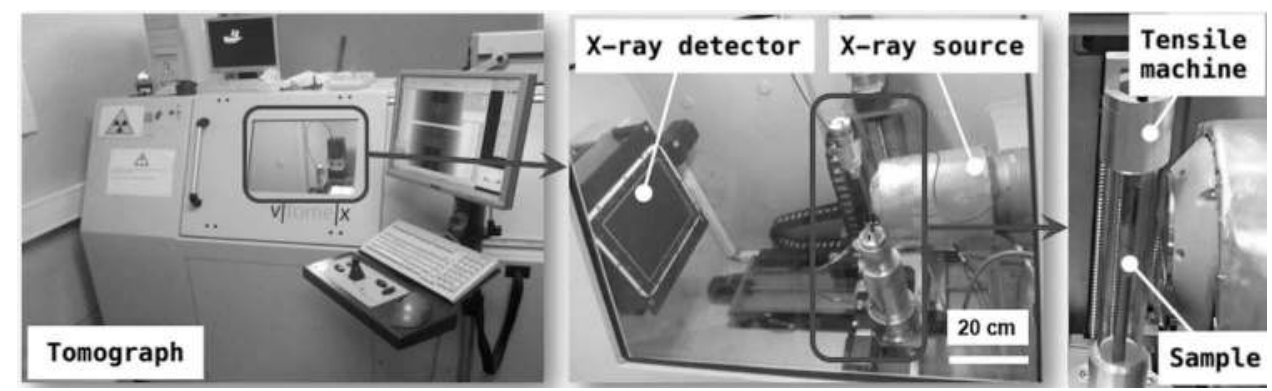

Figure 5. Experimental setup. ${ }^{[22]}$

The acquisition is performed for 1200 angular positions (Figure 4), for a total duration of approximately 10 minutes, with an exposure time of $500 \mathrm{~ms}$ for each projection, and an X-ray source operated with a voltage of $80 \mathrm{kV}$ and a current of $280 \mu \mathrm{A}$. The size of the reconstructed volume is $1500 \times 1500 \times 500$ voxels. Examples of tomographic slices may be found in Figure 6.

\section{Analysis technique}

\subsection{Preliminary discussion}

In order to exploit the data from the reconstructed volumes, it is necessary to perform a segmentation, in order to isolate the different phases. It is a common concern in the image processing and computer vision fields, and it has been the object of many studies, which suggested a large variety of methodologies to answer this issue. ${ }^{[23]}$

The easiest way to segment biphasic greyscale data is to use a threshold: a greyscale level (i.e. the threshold) is chosen, and the pixels of the image are 


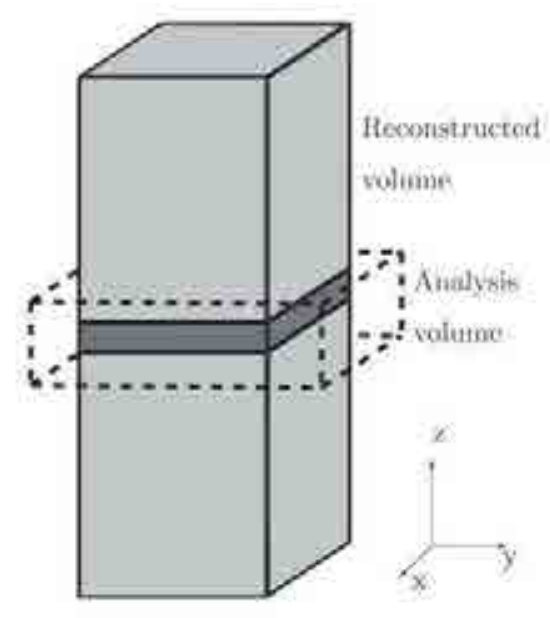

(a) Region of interest of the mini-Scarf samples

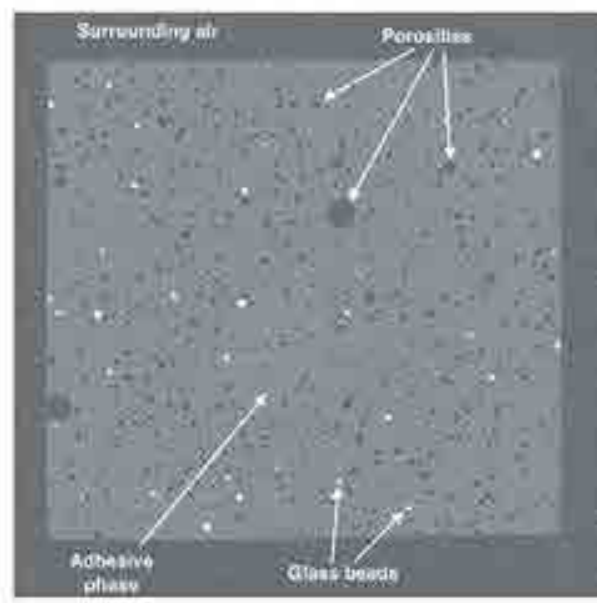

(b) CT-scan after reconstruction (adhesive B, $X Y$ plane)

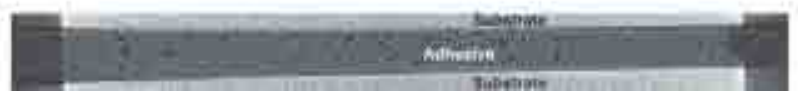

(c) CT-scan after reconstruction (adhesive $\mathrm{A}, X Z$ plane)

Figure 6. Reconstructed data after filtering and cropping.

separated whether they are brighter or darker than this value. Even though this method is fairly basic, it usually gives satisfactory results, given the fact that the data histogram is adequate (i.e. that the phases are easily distinguishable). The threshold can be manually chosen or algorithmically computed, the latter being preferable. The numerical determination of the threshold can be performed in various ways: one may base the calculation upon the analysis of the histogram, ${ }^{[24]}$ or by clustering the greyscale levels, ${ }^{[25]}$ for instance. The thoroughly documented paper of Sezguin et al. ${ }^{[23]}$ compiles an extensive list of methodologies and should be consulted for advanced details. Other methods, not necessarily based on thresholding, are available, but they will not be discussed here, as it is out of the scope of this paper.

Due to the shape of the histogram in Figure $7 \mathrm{a}$, an Otsu thresholding ${ }^{[25]}$ is used. In this paper, Otsu demonstrates that it is possible to compute an optimal threshold to segment bimodal data by maximising the interclass variance of the greyscale levels in each class. It is though necessary to generalise this approach to a thresholds number $N_{\text {thr }}$ greater than 1, as Figure 7a displays a trimodal histogram. Otsu suggests that it is easily performed, by definition of the discriminant criterion chosen to optimise the thresholds values, ${ }^{[25]}$ upon which there is no constraint regarding $N_{t h r}$. In this study, a multithresholding process is used for $N_{t h r}=2$, in order to discriminate the following phases: (i) the pores and the surrounding air in the tomograph chamber, (ii) the adhesive matrix, and (iii) the substrates (added to the glass beads, depending on the adhesive). It should 


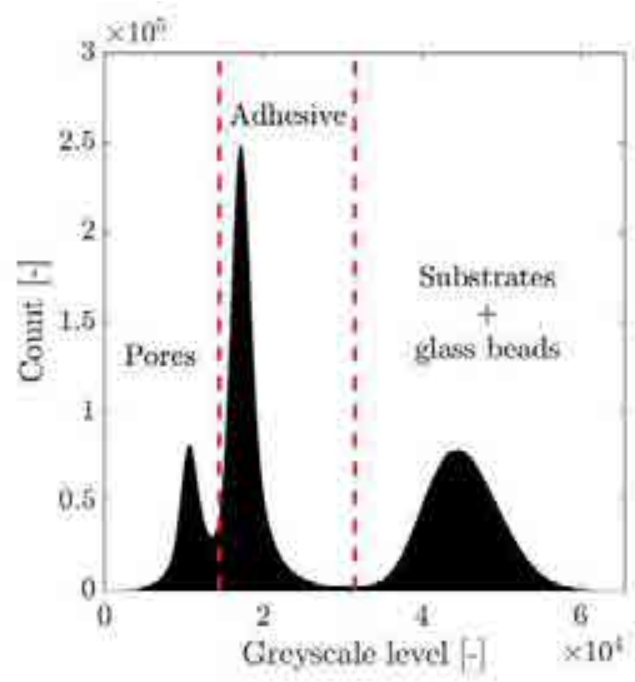

(a) Histogram of a reconstructed volume and thresholds computed using Otsu's method

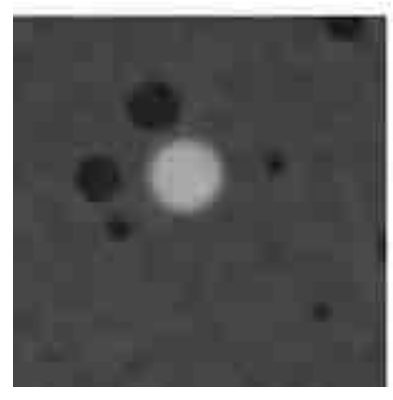

(b) Example of raw volume data

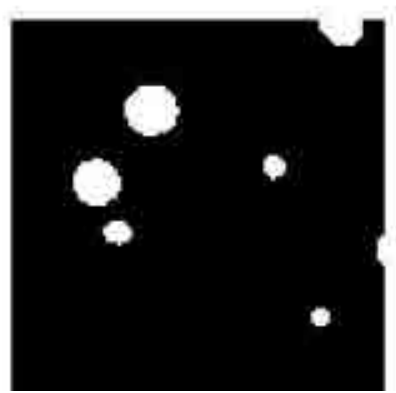

(c) Pixels below the first threshold (i.e. pores, )

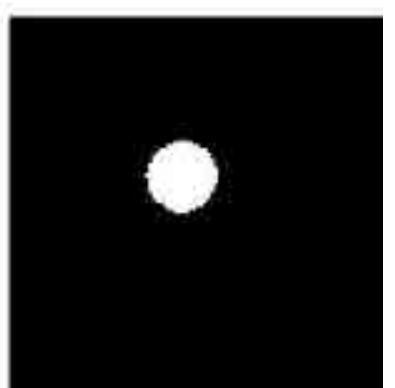

(d) Pixels above the second threshold (i.e. glass beads,)

Figure 7. Segmentation principle.

also be noted that no significant change in illumination for the same phase has been experienced, which could have prevented us from using such a method.

It is then obvious that the weak spot of the chosen methodology is the sensitivity to the noise in the experimental data. In order to minimise the influence of this measurement noise, it is then advised to filter the data in order to increase as much as possible the signal-to-noise ratio. It is however paramount not to damage the data, and especially the contours of the objects that must be segmented (i.e. the pores). A great number of filters (twodimensional or three-dimensional) are available. ${ }^{[26]}$ Among all these possibilities, the median filter and its derivates stand out with their edges preservation properties, as it was proven by Gallagher et al. ${ }^{[27]}$ The filter used for the works presented in this paper is a three-dimensional median filter, whose kernel size will be discussed further. 


\subsection{Preprocessing}

As mentioned previously, the reconstructed data need to be filtered, using a $3 \mathrm{D}$ median filter. The size of the kernel of the median filter will determine the minimal size of the segmented entities to consider. The filtering is performed with Matlab. A kernel size of 5 voxels is chosen, to remain relatively small when compared to the majority of the pores. As a consequence, any segmented pore whose volume is lesser than 125 voxels (i.e. the volume of the kernel) will be considered as too uncertain, and therefore will be discarded. The chosen filter is three-dimensional to prevent as much as possible the loss of information during the filtering.

The shape of the reconstructed volumes is a $(1500,1500,500)$ matrix. They are cropped to keep only the region of interest (the adhesive joint and a small part of the substrates, Figure 6a).

\subsection{Segmentation}

As stated in preamble, the segmentation is performed using Otsu algorithm ${ }^{[25]}$ to compute the required thresholds. The greyscale values of these thresholds are hence unsupervisedly computed, using the histogram of the data and the statistics of the greyscale levels. Such data display well-defined peaks and valleys in-between (in Figure 7a, from left to right, pores, adhesive, and substrates and beads which show roughly the same attenuation coefficient), enabling an histogram-based segmentation method. The computed thresholds may be visualised on the histogram in Figure 7a, which is provided as an example. One may notice that the lowest threshold does not exactly fit the minimum between the two corresponding peaks in Figure 7a, which is rather counter-intuitive. This is due to the slight asymmetry in terms of measurement noise, as it will be addressed later in the study.

Using these thresholds, it is then possible to build volumes containing only the identified phases so far: pores + surrounding air, adhesive, and substrates + beads.

These volumes need to be filtered either to remove unwanted objects (the air surrounding the sample in the tomograph chamber, the measurements artefacts which may occur near the interfaces and the objects whose size is too small to be taken into account) or to separate different objects that were placed in the same phase (typically, the glass beads if the adhesive contains some and the aluminium substrates). This step is easily completed by considering the sizes of all these items, to identify those that should be discarded. For instance, the air surrounding the sample features a volume significantly larger than any other pore in the adhesive joint, and thus may be easily labelled in the segmented volume to be erased. The same method may also be used to classify the objects contained in the (substrates + glass beads) phase since the substrates volumes are several orders of magnitude greater than the volume of a glass bead. 
Before being used on the experimental data, this tool needs to be characterised on synthetic representative data, in order to validate its architecture and to investigate its limitations.

\section{Characterisation of the analysis tool}

\subsection{Creation of synthetic volumes}

In order to validate the methodology proposed in this paper, it was important to verify the segmentation performances of the tool on synthetic data. These artificial datasets should be representative of the experimental data in terms of (i) greyscale levels, (ii) noise, and (iii) pores sizes. The diameters of the pores are assumed to be following a Gaussian distribution, whose parameters will be discussed later. Their centroids are assumed to be randomly distributed within the adhesive joint. This hypothesis is validated further in this paper.

The creation of such a volume is divided into four steps. Firstly, a reference boolean volume is created, where true values denote the presence of pores pixels, and false values denote the absence of pores. Secondly, this boolean volume is turned into a 16-bits greyscale volume, where the pores display a unique greyscale level, and where the adhesive displays another, distinct, greyscale level. Then, padding is added (i.e. the air surrounding the sample and the substrates), since it influences the shape of the histogram of the data. Lastly, Gaussian noise is added to the synthetic volume, and a slight blurring of the contours is applied in order to best resemble the experimental data. The process adopted to create these synthetic volumes is summarised in Figure $8(a, b)$.

\subsubsection{On the boolean reference generation}

A representative volume of $[300,300,100]$ voxels for the adhesive joint is used in the following considerations. The complete synthetic volume will be slightly larger, due to the padding operated in the following steps, as presented in Figure 8b. The pores diameters follow a Gaussian distribution whose mean and standard deviation are, respectively, $\mu_{d}$ and $\sigma_{d}$. The study is done for 200 pores randomly located in the adhesive joint. The boolean reference is under the shape of a matrix $[300,300,100] M^{\text {ref }}$, whose elements $(i, j, k)$ are defined by Eq. 1 .

$$
\left\{\begin{array}{l}
M_{i j k}^{r e f}=1, \text { if } \sqrt{\left(i-x_{c}\right)^{2}+\left(j-y_{c}\right)^{2}+\left(k-z_{c}\right)^{2}} \leq r_{p o r} \\
M_{i j k}^{r e f}=0 \text { else }
\end{array}\right.
$$

where $r_{p o r}$ is the radius of the considered pore, and $\left(x_{c}, y_{c}, z_{c}\right)$ the coordinates of the centre of this pore. As a side note, the properties of the pores are not constrained to be integers (i.e. the quadruplet $\left.\left(x_{c}, y_{c}, z_{c}, r_{\text {por }}\right) \in \mathbb{R}^{4}\right)$. 


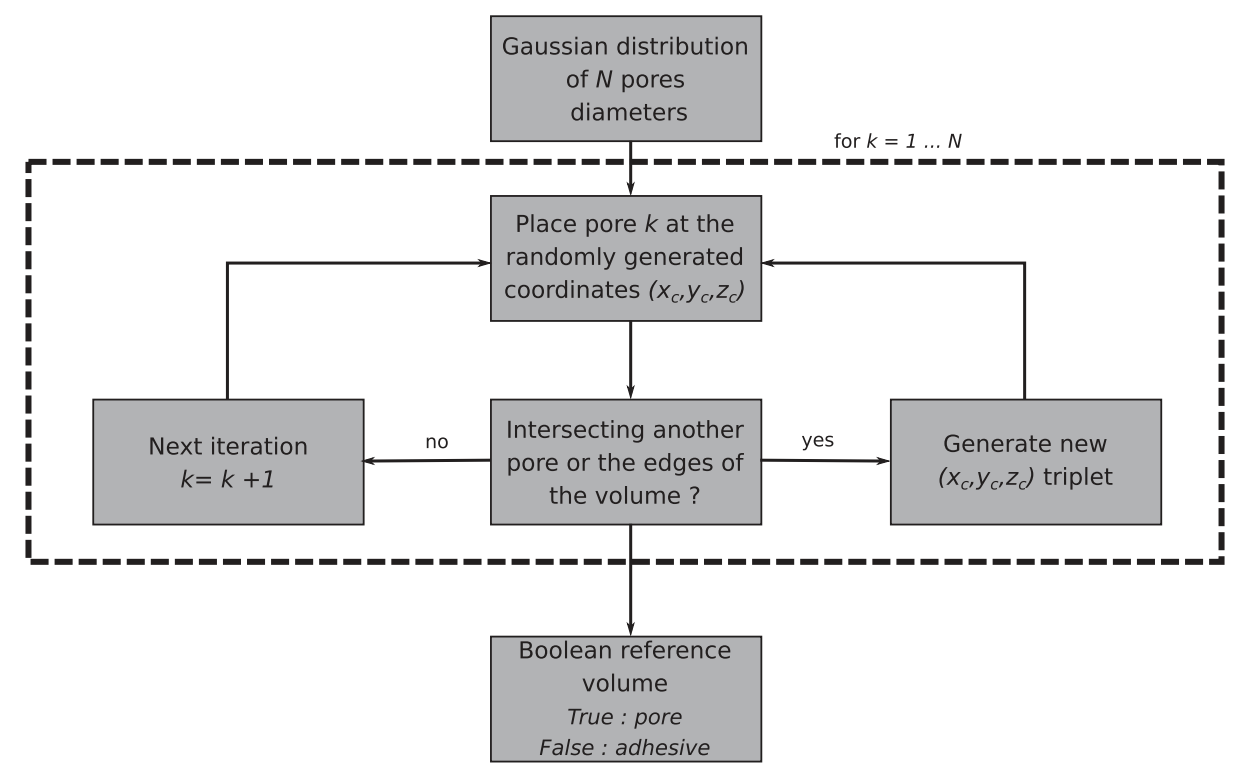

(a) Creation of a boolean reference volume, to be used as a template for a synthetic greyscale volume

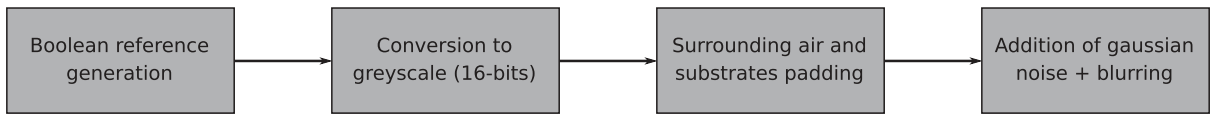

(b) Process adopted to create synthetic microtomographic reconstructed data from a boolean reference

Figure 8. Generation of synthetic data to characterise the performances of the proposed analysis tool.

The process mathematically expressed in Eq.1 also simulates the voxelisation which occurs during the microtomography measurements, with the discretisation of continuous quantities (i.e. the material) by the X-ray detector.

It is conspicuous that the parameters chosen for the diameters Gaussian distribution will play an important part on the segmentation performances of the designed tool: the smaller the generated pores, the poorer the tool will compare to the reference. The values of these $\left[\mu_{d}, \sigma_{d}\right]$ parameters will be discussed later in the study.

\subsubsection{On the greyscale levels of the different phases}

The determination of the greyscale values to be used for each phase is straight-forward, as the histograms of the reconstructed volumes are provided. The nominal greyscale value for each synthetic phase is the peak value evaluated on the histograms of the experimental data (see Figure $7 \mathrm{a}$ for example). The greyscale values (before the addition of the Gaussian noise, see in Figure $8 \mathrm{~b}$ ) are summarised in Table 3. 
Table 3. 16-bits nominal greyscale values for each phase in the investigated adhesives.

\begin{tabular}{lcc}
\hline Phase & Adhesive A & Adhesive B \\
\hline Pores + ambient air & 10407 & 10563 \\
Adhesive material & 18250 & 17220 \\
Substrates (+ glass beads if needed) & 44980 & 44700 \\
\hline
\end{tabular}

\subsubsection{On measurement noise}

The measurement noise is estimated by acquiring data for the same volume twice, and by subtracting one of the resulting reconstructed volumes to the other. The remaining greyscale values correspond to the measurement noise caused by the X-ray detector. A significantly higher noise for the substrates and the glass beads is expected due to their higher X-ray linear attenuation coefficient $\mu$. The noise values for each phase are listed in Table 4.

A light Gaussian filter ( $\sigma=0.4$ voxel) is applied in order to slightly blur the edges of the pores to replicate the experimental data. The comparison between the histograms of the simulated data and of the experimental data should provide a good indicator of the quality of the synthetic volumes, and of their representativeness of the actual microtomographic data (Figure 9). The data represented in Figure 9 have been normalised with respect to the total numbers of pixels in each case (synthetic data and experimental data), and therefore they are expressed in terms of frequencies.

A few remarks should be made at this point of the study: (i) one can see a slight mismatch in terms of frequencies for each peak, certainly due to the padding added during the creation of the synthetic volume; (ii) the in-between zone for the pores and adhesives peaks is seemingly representative of the real data; (iii) the brightest pixels for the synthetic adhesive (greyscale levels between 20000 and 30000, Figure 9) are lacking a few occurrences to match the experimental frequencies. Due to the large difference between the peak values for the adhesive pixels and for the substrates pixels, this particular mismatch should not impact too much the computation of the corresponding threshold. Moreover, the pixels of the substrates in this in-between zone are mostly located within the bulk of the aluminium (visible in Figure 6c), meaning that segmentation errors potentially occurring in these area can easily be fixed by morphological operations if need be (i.e. opening, closing, filling, etc.). Alternatively, this mismatch (due to the fact that each phase does not follow a perfectly symmetrical Gaussian distribution) could be corrected by stretching the greyscale levels in this interval

Table 4. 16-bits noise greyscale values for each phase in the investigated adhesives.

\begin{tabular}{lcc}
\hline Phase & Adhesive A & Adhesive B \\
\hline Pores + surrounding air & 2281 & 2254 \\
Adhesive material & 2554 & 2462 \\
Substrates (+ glass beads if needed) & 6586 & 6681 \\
\hline
\end{tabular}




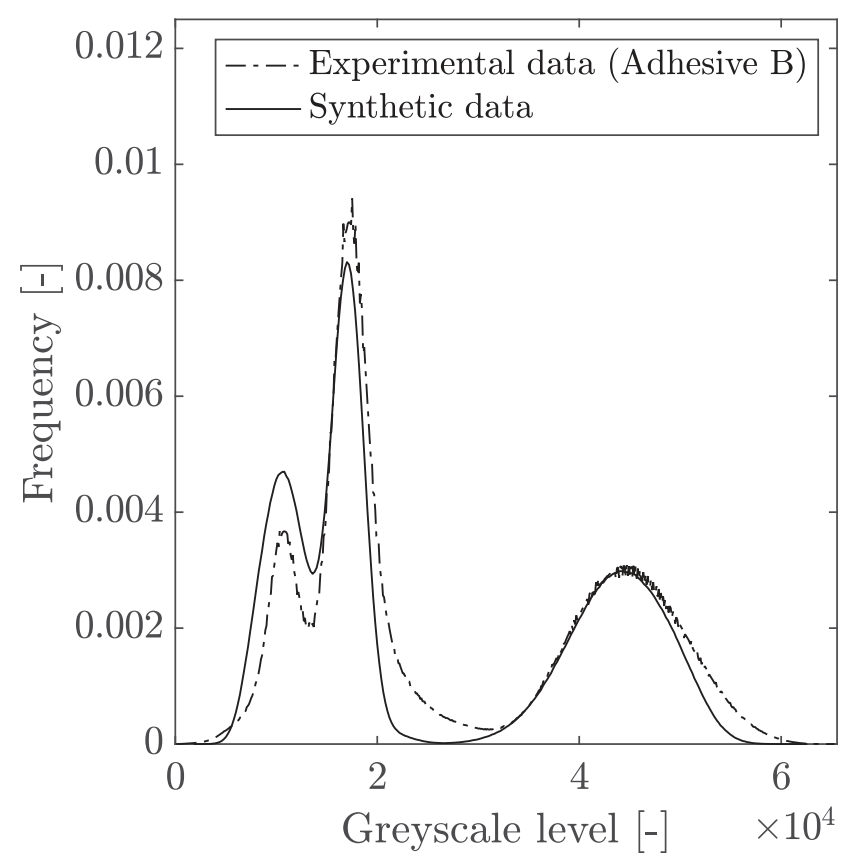

Figure 9. Comparing the histogram of synthetic data with the histogram of experimental data (adhesive B).

by a multiplicative factor until the synthetic histogram matches the experimental histogram. It is however not performed in this study as it is assumed to be a second-order factor on the segmentation abilities of the developed tool. Another indicator of the representativeness of the simulated volume is the similarity between the thresholds computed for the synthetic data and the ones computed for the experimental volumes, which were used as a reference pattern to build the artificial data. A comparison is made for the two adhesives studied in this paper in Table 5, where the first (pores/adhesive) and second (adhesive/substrates) thresholds values are provided.

\subsection{Segmentation abilities}

The segmentation tool is then applied to these synthetic volumes, in order to compare the segmented data with the reference pores patterns (Figure 8a). As it has been evoked earlier in this paper, significant variations are expected in terms of performances depending on the size of the pores to be segmented. Various diameters distributions are therefore considered, included in several simulated volumes as detailed previously. In practice, the parameters of the diameters Gaussian distribution $\left(\left[\mu_{d}, \sigma_{d}\right]\right)$ will be incrementally increased in two intervals, in order to sweep a matrix of values for this couple. The mean value $\mu_{d}$ will be varied from 8 to 20 voxels, and the standard deviation $\sigma_{d}$ will be varied from 1 to 5 voxels. Due to the similarity in terms of greyscale levels between the adhesive 
Table 5. Comparison between the experimental and synthetic 16-bits greyscale thresholds for adhesive A and B.

\begin{tabular}{lcc}
\hline Thresholds & Adhesive A & Adhesive B \\
\hline First threshold (exp.) & 15009 & 14035 \\
First threshold (synth.) & 14151 & 13781 \\
Second threshold (exp.) & 32192 & 31531 \\
Second threshold (synth.) & 31085 & 30466 \\
\hline
\end{tabular}

A and the adhesive B (see Tables 3, 4 and 5), the study will be carried out only for one adhesive (adhesive B). For each couple $\left[\mu_{d}, \sigma_{d}\right]$, a relative error $\varepsilon$ for the pores number and the pores ratio with respect to the references values will be calculated, as shown in Eqs.2 and 3. It should be noted that these errors are expressed as percentages, due to the normalisation with respect to $N_{p o r}^{\text {ref }}$ and $\eta_{p o r}^{\text {ref }}$ in Eqs. 2 and 3.

$$
\begin{gathered}
\varepsilon_{N}=100 * \frac{\sqrt{\left(N_{p o r}^{\text {det }}-N_{\text {por }}^{r e f}\right)^{2}}}{N_{\text {por }}^{\text {ref }}} \\
\varepsilon_{\eta}=100 * \frac{\sqrt{\left(\eta_{\text {por }}^{\text {det }}-\eta_{\text {por }}^{r e f}\right)^{2}}}{\eta_{\text {por }}^{\text {ref }}} \text {, with } \eta_{\text {por }}=\frac{V_{\text {por }}}{V_{\text {joint }}}
\end{gathered}
$$

where $N_{\text {por }}^{\text {det }}$ and $N_{\text {por }}^{\text {ref }}$ are, respectively, the numbers of pores detected and of reference, $\eta_{\text {por }}^{\text {det }}$ and $\eta_{\text {por }}^{\text {ref }}$ are, respectively, the detected and reference pores volumetric ratio, and $V_{\text {por }}$ and $V_{\text {joint }}$ are, respectively, the volumes of the pores and of the joint. In order to reduce the influence of the greyscale noise on the results, 5 computations will be carried out for each couple $\left[\mu_{d}, \sigma_{d}\right]$ for different Gaussian noise draws (the standard deviation of this noise being tuned using the data displayed in Table 4). The results of this parametric study are displayed in Figure 10, where the mean values for $\varepsilon_{N}$ and $\varepsilon_{\eta}$ are presented.

Apart from the extreme cases of small pores distributions, satisfactory results are obtained (typically, for $\mu_{d} \leq 10$ voxels), as the relative errors $\varepsilon$ remain circa $5 \%$ or below. The major part of the segmentation errors are expected to happen near the edges of the pores, due to the slight blurring occurring at these locations.

These results on synthetic volumes ensure us that once applied on the experimental data, our method should yield fairly satisfying results, to be pondered with the size of the segmented pores, which was labelled as an important factor in terms of results accuracy. 


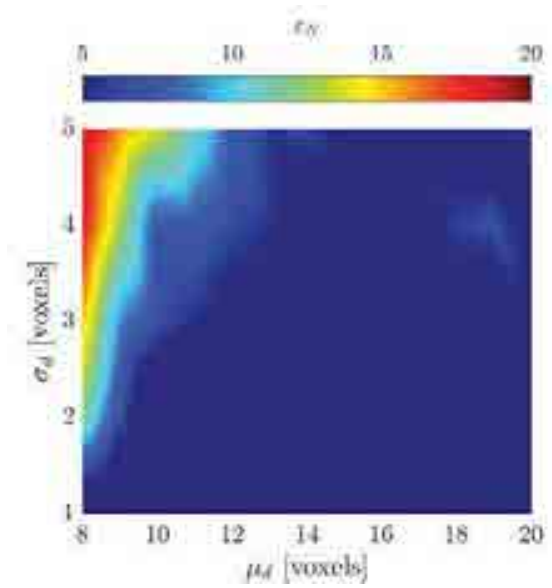

(a) Mean value of the relative error on the detected pores number $\varepsilon_{N}$

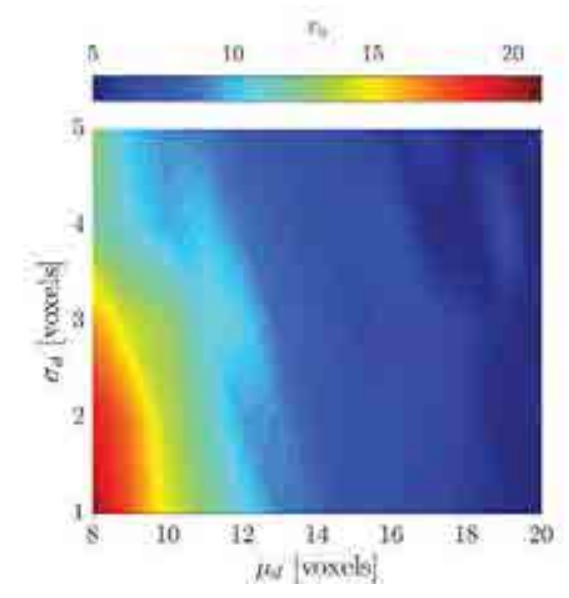

(b) Mean value of the relative error on the detected pores volume ratio $\varepsilon_{\eta}$

Figure 10. Segmentation performances on the pores numbers and volume ratio as functions of $\left[\mu_{d}, \sigma_{d}\right]$.

\section{Results}

Data similar to Figure 11(b,d) are obtained once the segmentation process is completed. It is then possible to carry out various considerations on these datasets, whether geometrical (volume, shape, equivalent diameter, etc.) or microstructural (pore-to-joint volumetric ratio, pores number, etc.).

A 3D view of a reconstructed sample computed with FiJi software ${ }^{[28]}$ can be seen in Figure 12. The Cartesian coordinate system defined in this figure is used in the following sections to express the location of the pores. As one may notice, the surfaces of the substrates are not perfectly parallel to one another. This is most certainly caused by the machining of the substrates, the mechanical treatment applied to the bonding surfaces and more generally an imperfect bonding process. This misalignment, however, does not disturb the observations made in this study.

\subsection{Pores numbers, volumetric fractions, and diameters distributions}

The X-ray microtomography measurements data are segmented (see Figure 13 for $3 \mathrm{D}$ viewing) using the previously detailed methodology in their initial state. Two different curing cycles are then to be investigated, for two different adhesives. As a reminder, the studied curing cycles are the following: (i) $110^{\circ} \mathrm{C}$ for $1 \mathrm{~h} 10$, hereafter referred to as "high temperature" and (ii) $23^{\circ} \mathrm{C}$ for $24 \mathrm{~h}+80^{\circ} \mathrm{C}$ for $2 \mathrm{~h}$, hereafter referred to "low temperature". For each case, it is possible to compute the number of pores included in the adhesive joint, and the volumetric fraction of these pores with respect to the whole joint. These results are shown in Figure $14(\mathrm{c}, \mathrm{d})$. The uncertainty on the considered quantities ( $c$. the error bars in 


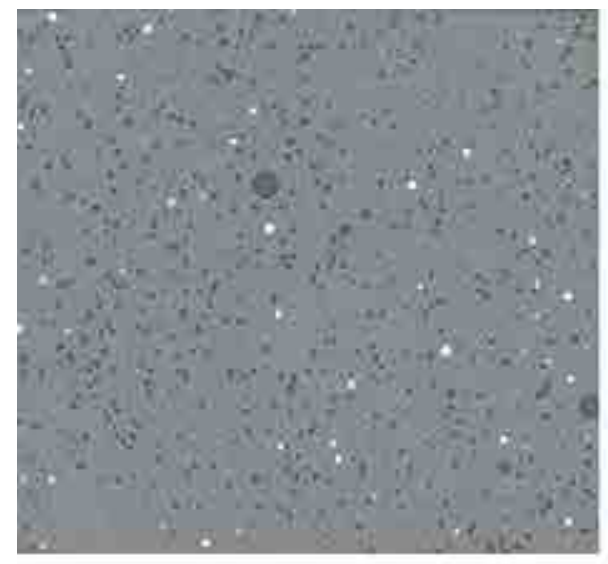

(a) Raw data

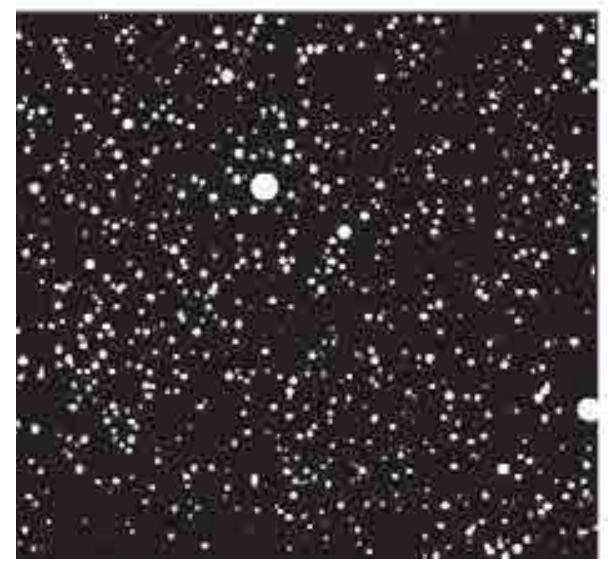

(c) Pores (white pixels)

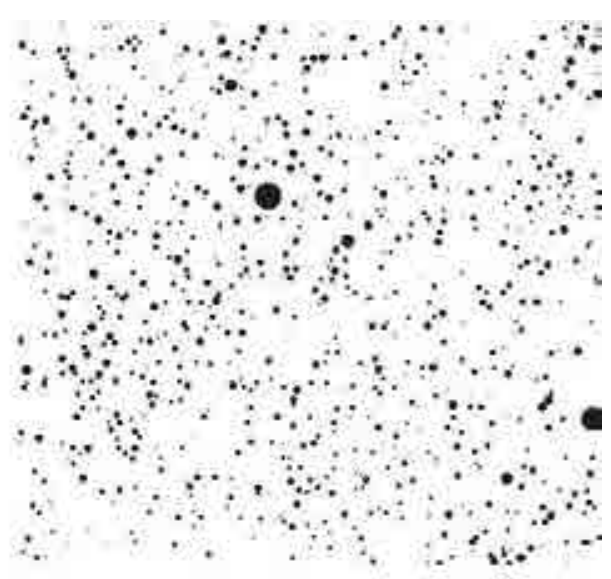

(b) Adhesive (white pixels)

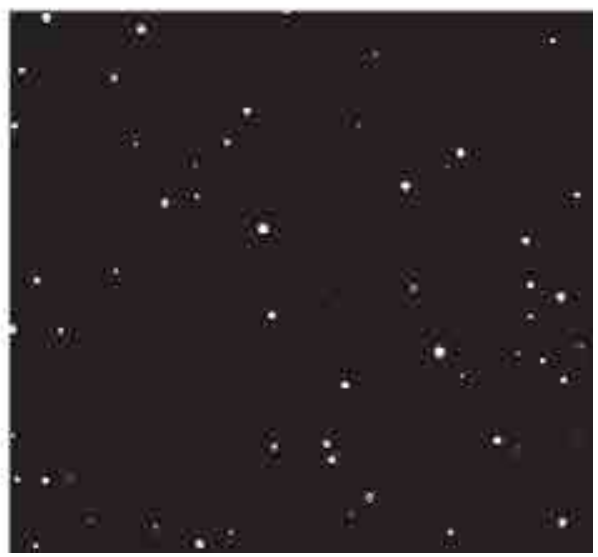

(d) Glass beads (white pixels)

Figure 11. Segmentation step results (adhesive B, slice in the middle of the adhesive joint).

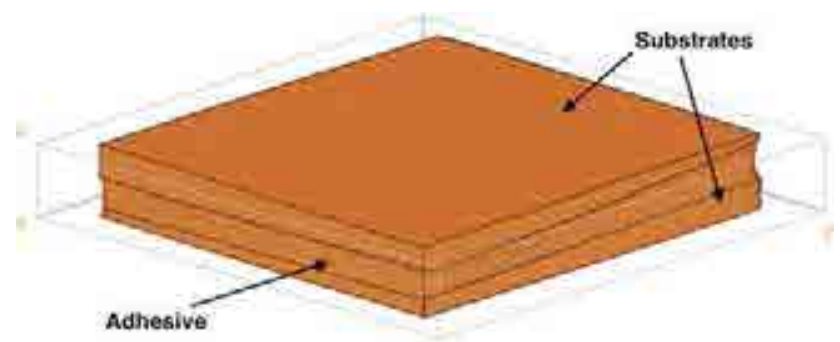

Figure 12. 3D view of a reconstructed sample (outlined contours for better visualisation).

Figure 14(c.d)) was estimated by cross-referencing the data in Table 7 and in Figure 10. It is also possible to compute the distributions of the equivalent diameters of the pores for each case, as it is done in Figure 14(a,b). The pores count in each bin in these histograms is normalised with respect to the total 


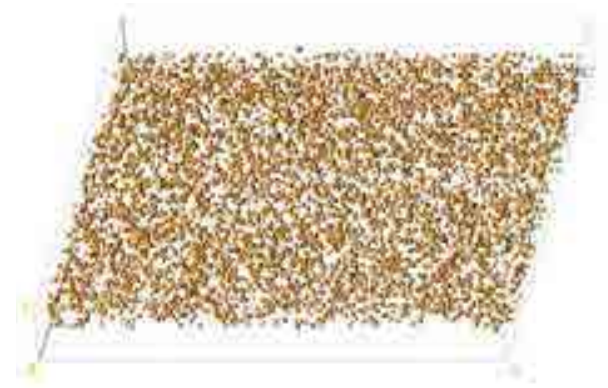

(a) Adhesive A, low temperature curing

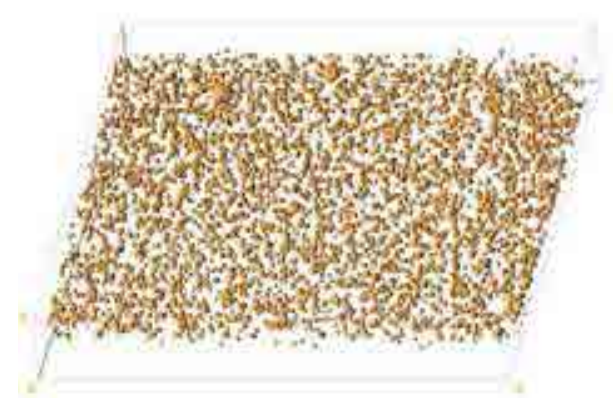

(c) Adhesive B, low temperature curing

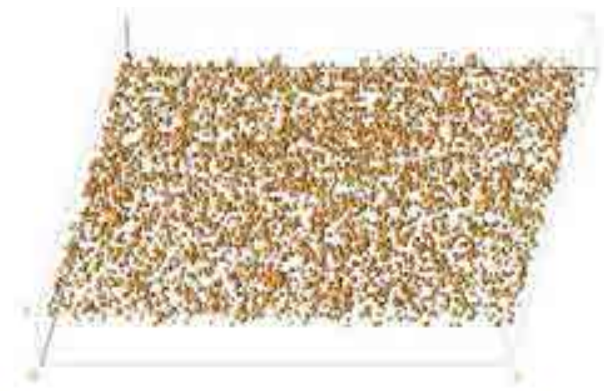

(b) Adhesive A, high temperature curing

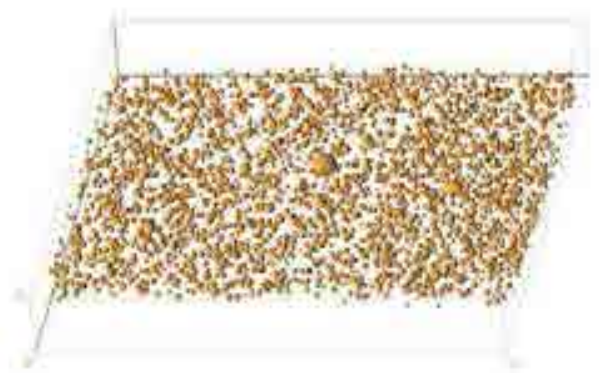

(d) Adhesive B, high temperature curing

Figure 13. 3D views of segmented pores in adhesive joints.

number of pores, in order to obtain frequencies histograms, in order to easily compare the different samples, each one containing a different number of pores.

From the data displayed in Figure 14, it appears that the two adhesives investigated in this paper feature different sensitivities to the curing conditions, at least for the considered thermal cycles examined in the present study.

On one hand, the adhesive A seems rather unresponsive to the curing temperature: very little changes can be identified between high temperature curing and low temperature curing, in terms of pores number (Figure 14c), porosity volume ratio (Figure 14d) and diameters distribution (Figure 14a). Detailed numerical values may be found in Table 6.

On the other hand, the adhesive B is far more impacted by an increased curing temperature, as it may be seen in Figure 14(b,d). From these data, one may notice that a higher polymerisation temperature for the adhesive $\mathrm{B}$ translates into a slightly higher average pore diameter (from $43 \mu \mathrm{m}$ to $50 \mu \mathrm{m}$, Table 7).

A possible conclusion to these findings could be that the high temperature cycle tends to trigger void growth phenomena, either relying on material expansion, or on coalescence mechanisms possibly activated by the thermal environment. ${ }^{[15]}$ Another finding of these initial state measurements is that, no matter the curing cycle, the microstructural properties of the adhesive A and those of the adhesive B are extremely different (see Figures 14a and 14a), even though they are similar materials: both are bicomponent epoxy 


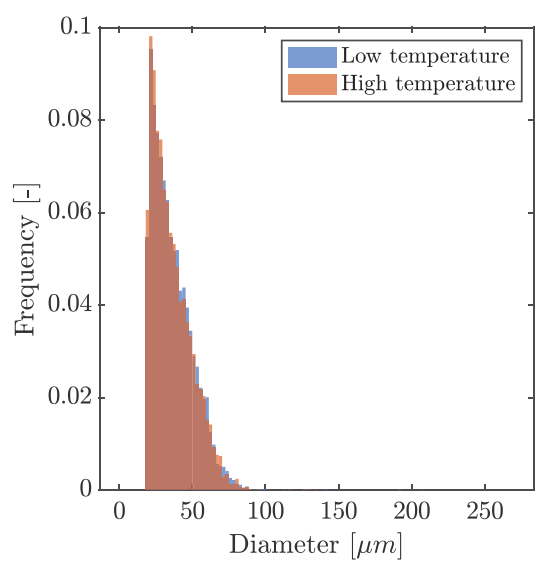

(a) Influence of the curing cycle on the pores diameters, adhesive $\mathrm{A}$

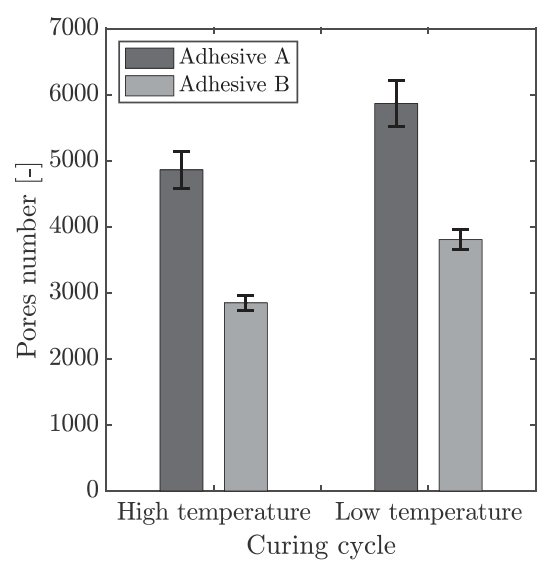

(c) Influence of the curing cycle on the pores number

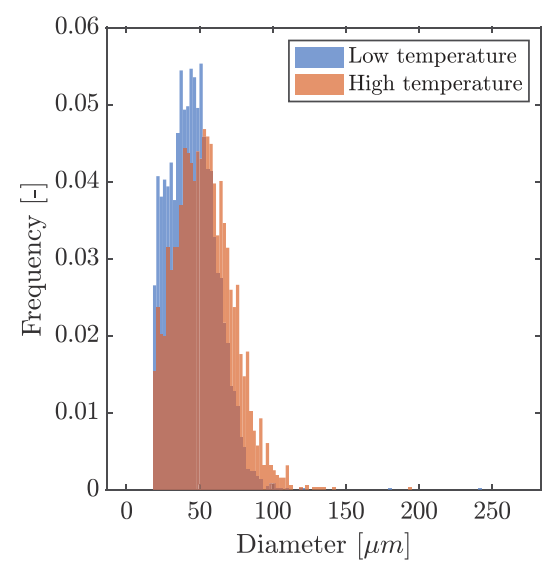

(b) Influence of the curing cycle on the pores diameters, adhesive B

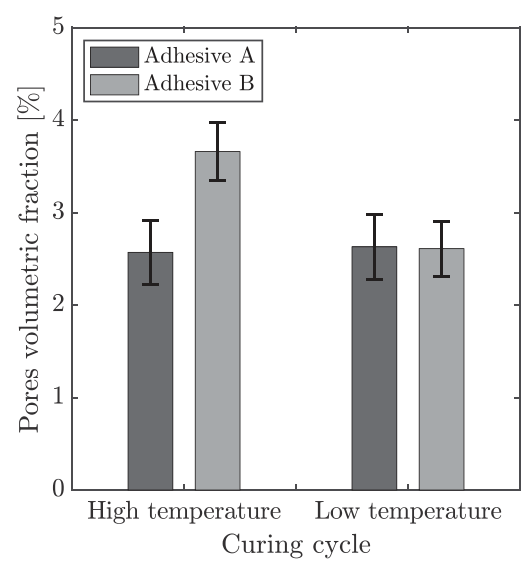

(d) Influence of the curing cycle on the pores volumetric fraction

Figure 14. Comparisons of the pores diameters, numbers, and volumetric fractions for high and low temperatures curing cycles.

Table 6. Characteristics of the created pores in the adhesives A and B for different curing cycles.

\begin{tabular}{llcc}
\hline Adhesive & Curing & Pores [-] & Pores ratio [\%] \\
\hline A & Low temperature & 5866 & 2.63 \\
A & High temperature & 4862 & 2.57 \\
B & Low temperature & 3806 & 2.61 \\
B & High temperature & 2849 & 3.67 \\
\hline
\end{tabular}

structural adhesives, with similar mechanical properties. The major differences between the two considered materials (aside from their chemical compositions, as they are not available) are shown in Table 1. 
Table 7. Pores diameters statistics for all the considered cases.

\begin{tabular}{llcc}
\hline Adhesive & Curing & Mean diameter $[\mu \mathrm{m}]$ & Standard deviation $[\mu \mathrm{m}]$ \\
\hline A & Low temperature & 43.1 & 12.0 \\
A & High temperature & 42.9 & 11.9 \\
B & Low temperature & 49.5 & 14.1 \\
B & High temperature & 56.3 & 18.2 \\
\hline
\end{tabular}

As a reminder, it was evoked earlier in this paper that two additional specimens were studied for the following cases: adhesive A cured at low temperature and adhesive B cured at high temperature. Given for informative purposes only regarding the experimental scattering, these samples displayed the following properties, respectively: $2.63 \%$ pores ratio with 5828 pores, and $3.87 \%$ pores ratio with 3108 pores.

\subsection{Pores shape}

From the gathered data (Figure 2a,d, 6b, $6 \mathrm{c}$ and 11, for example), the pores in the adhesives are assumed to be rather spherical. It is however possible to quantify their roundness and their resemblance to a sphere. A quantity useful for such considerations is the sphericity, defined by Wadell in $1935 .{ }^{[29]}$ The sphericity $\Psi$ of a particle of volume $V_{p}$ and of area $A_{p}$ is defined according to Eq.4.

$$
\Psi=\frac{\pi^{\frac{1}{3}}\left(6 V_{p}\right)^{\frac{2}{3}}}{A_{p}}
$$

In the case of a perfect sphere, $\Psi=1$. Thus, the closer to 1 is the sphericity, the more spherical the considered object is. Using connected components analysis on the segmented pores volumes, it is possible to extract, for each pore, its volume and its surface, and to use the expression in Eq.4 to compute its sphericity. The data plotted in Figure 15 represent these sphericities with respect to the equivalent diameters of the pores for all the considered cases.

It is fairly obvious from Figure $15(a, b)$ that the majority of the detected pores are almost perfectly spherical, since for both the adhesives and for both the curing cycles, there are clusters of point located near $\Psi=1$. This assertion is confirmed by the data in Figure 15(c,d), where the distributions of the sphericities values are presented. These distributions are normalised with respect to the total number of pores, yielding frequencies instead of counts for each interval in the histograms. One may clearly see that the vast majority of these values are close to $\Psi=1$.

The definition of the sphericity introduced by Wadell ${ }^{[29]}$ assumes that $\Psi=1$ is a theoretical limit for $\Psi$. However, values slightly greater than 1 may be noticed in Figure $(15 \mathrm{a}, \mathrm{d})$. The fact that these paradoxical values occur for small diameters tends to suggest that it may be an effect of the voxelisation, 


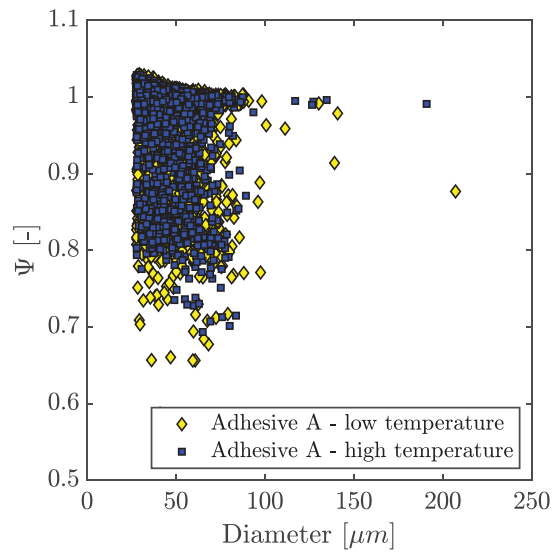

(a) Sphericitiy of the pores in adhesive A plotted with respect to their diameter

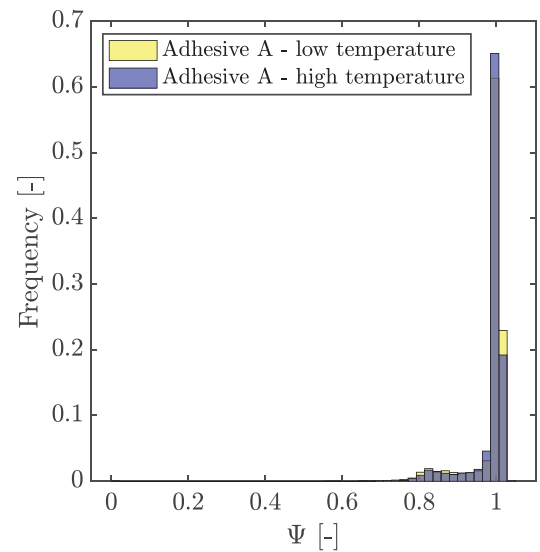

(c) Sphericity values distribution for the adhesive A

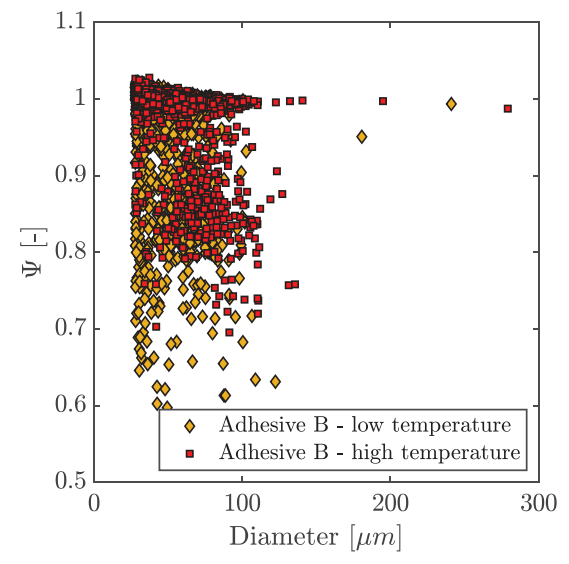

(b) Sphericity of the pores in adhesive B plotted with respect to their diameter

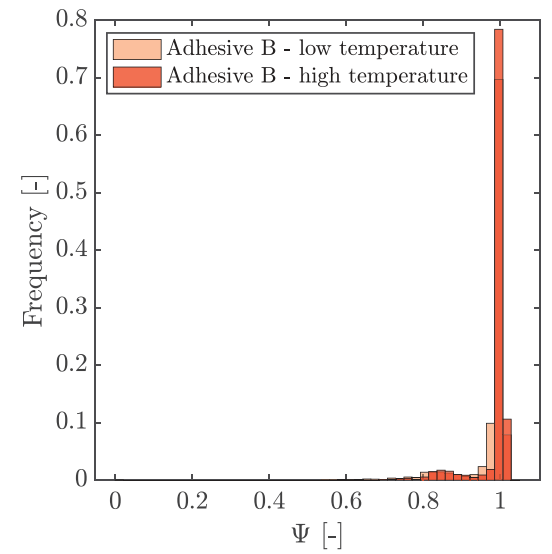

(d) Sphericity values distribution for the adhesive B

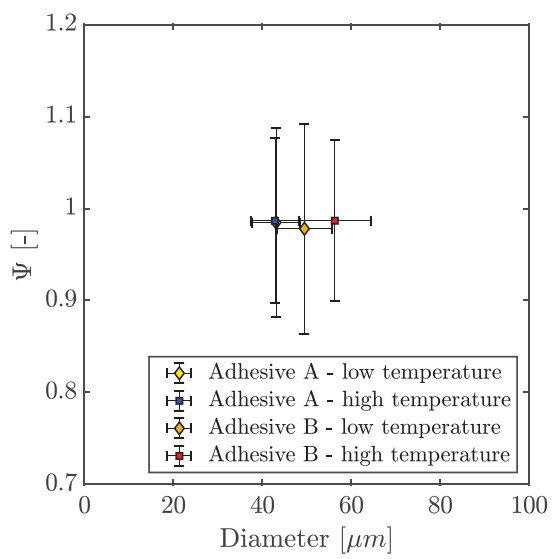

(e) Average sphericities for all the considered cases

Figure 15. Sphericities of the created pores. 
leading to slightly erroneous areas or volumes calculations. It is easy to understand that the smaller the size of the pore, the greater the effect of voxelisation, and therefore the greater the potential error on the sphericity.

One may also notice a few sphericity values rather small when compared to the trend on each graph (see in Figure 15(a,d)), lesser than 0.7. These points may be due to coalescing pores or to segmentation errors for pores very close to one another (and mistakenly identified as one unique entity). Examples of these punctual values can be found in Figure 16, where pores with $\Psi<0.75$ are displayed. It is, however, arduous to determine whether these values are segmentation errors or whether they are due to actual coalescence phenomena occurring during the curing of the adhesive. For this reason, it was chosen not to disconnect them, even though several algorithms such as the watershed transform exist to answer similar issues.

No noticeable difference between the adhesives and between the curing cycles can be highlighted in terms of shape of the pores, though, as the points clouds displayed in Figure 15(a,b) feature highly similar shapes. This is put into perspective in Figure 15e, where the average points of each point clouds presented in Figure 15 $(\mathrm{a}, \mathrm{b})$ are plotted together. The error bars are computed using the standard deviations of the equivalent diameters and of the sphericities $( \pm 2 \sigma)$. Almost no gap between the adhesive A data can be spotted, which suggests that this particular adhesive is rather insensitive to the polymerisation conditions, at least with respect to the adhesive B. This conclusion should nonetheless be pondered by the fact that phenomena similar to the adhesive $\mathrm{B}$ may be happening in the adhesive $\mathrm{A}$, but at a lower scale, undetectable with the measurements performed in this configuration. A slight shift is however detectable for the adhesive B (Figure 15e), both in terms of diameter (which was expected, see the data in Table 7, for example) and in terms of sphericity. Several factors could explain this difference between these samples: (i) it could simply be due to the experimental discrepancy from one adhesive joint to another and therefore be unrelated to the curing conditions, or (ii) the dilation happening throughout the curing cycle due to the increase in temperature could counter the effect of the slight mechanical load applied to the Scarf samples in their bonding apparatus (visible in Figure 3b) in order to control the joint thickness. More advanced and extensive measurements are needed in order to validate this hypothesis or to develop another possible explanation of this phenomenon.

\subsection{Effective section of the adhesive joint}

One of the most critical effects of the presence of pores within an adhesive joint is the decrease in effective section. The effective section is defined in Eq.5 as the ratio between the surface of adhesive $S_{a d h}$ and the surface of joint (i.e. the sum of the surfaces of adhesive $S_{a d h}$, of pores $S_{\text {por }}$ and of glass beads $S_{\text {beads }}$ ). 

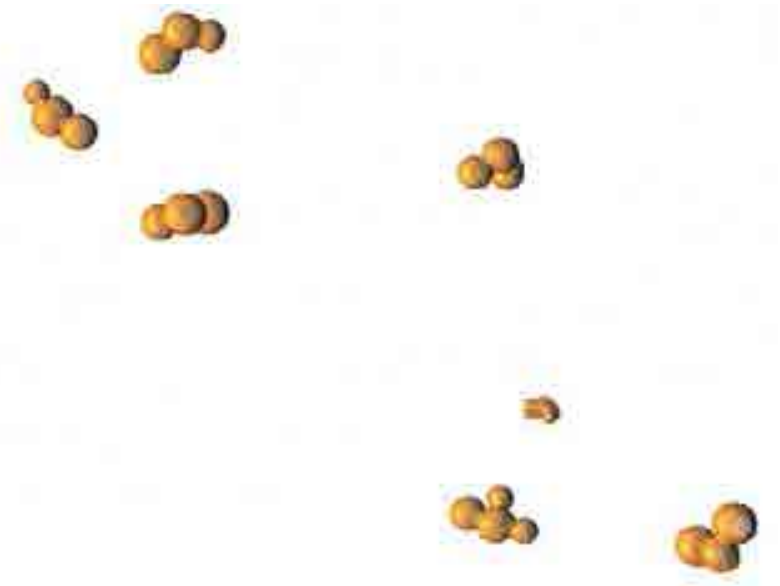

Figure 16. Merged pores inducing low sphericities (adhesive B, high temperature curing).

$$
\Sigma=\frac{S_{a d h}}{S_{a d h}+S_{\text {por }}+S_{\text {beads }}}
$$

This $\Sigma$ ratio (somehow similar to a surface ratio, by analogy with the volumetric ratio used earlier in our study) can then be computed for each slice of reconstructed volume containing adhesive (i.e. the slices where there is only aluminium are excluded). As the investigated bonded assemblies are designed for tensile loadings, the section planes are defined to be parallel to $(x y)$ in Figures $6 a$ and 12. It is then possible to compute $\Sigma$ with respect to the distance along $\vec{z}$ (defined in Figure 6a). It is assumed that $z=0$ for the first slice where adhesive is segmented.

Once again, the differences for the adhesive A are small for the two curing cycles studied in this paper. It should, however, be noticed that the pores seem to be concentrated at the center of the joint $(\sim z=0.2 \mathrm{~mm})$ for both cycles, with a sharper trend in the case of a high temperature curing. This remark is also to be made for the adhesive $B$, for which the differences between curing temperatures are once again more pronounced. If the rate of change of $\Sigma$ with respect to $z$ seems to be rather equivalent for both curves in Figure $17 \mathrm{~b}$ in the first and last quarters of the joint ( $z \leq 0.08 \mathrm{~mm}$ and $z \geq 0.3 \mathrm{~mm}$, roughly), it is however not the case in the middle: there is a sudden gap between low and high temperature curing, which could be explained by void growth patterns thermally activated during the polymerisation of the material. Such coalescence phenomena have been widely observed and studied for porous materials. The works of Pinzer ${ }^{[15]}$ in particular show that coalescence mechanisms can be thermally activated, which could explain the differences experienced between low temperature and high temperature curing for the adhesive B. This phenomenon is seemingly localised in the middle of the thickness of the joint, where the pores are more numerous. Additional work focused on the creation of the pores during the 


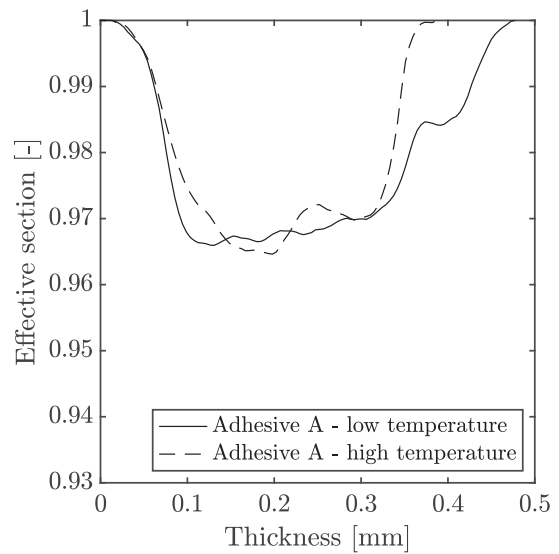

(a) Effective sections throughout the thickness of the adhesive joint (adhesive A)

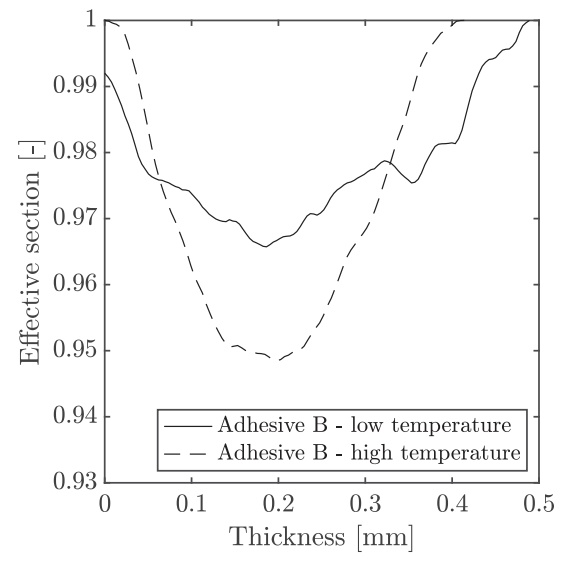

(b) Effective sections throughout the thickness of the adhesive joint (adhesive B)

Figure 17. Effective sections evolutions along the thickness of adhesive joints.

fabrication and the curing of the joint could bring elements to understand the mechanisms leading to the trends seen in Figure 17.

\subsection{Compactness}

Similar considerations can be pursued to investigate the existence of concentration zones for pores in the plane of the adhesive joint (as opposed to the data provided in Figure 17, where concentration zones along the direction normal to the adhesive joint were investigated). To do so, a similar approach is used, and define a compactness quantity $C$ computed along the $\vec{z}$ axis (see Figure 6a for axis definition). Therefore, this $C$ quantity will take the shape of a $[m, n]$ matrix for a given segmented adhesive phase and a given joint (adhesive+pores+beads) represented, respectively, by $[m, n, p]$ boolean matrices $M_{a d h}$ and $M_{\text {joint }}$, as it is expressed in Eq.6. The value of $C$ is an indicator of the effective thickness, by analogy with Figure 17 and its corresponding discussion.

$$
C(i, j)=\frac{\sum_{k=1}^{p} M_{a d h}(i, j, k)}{\sum_{k=1}^{p} M_{\text {joint }}(i, j, k)}
$$

The resulting maps of the compactness $C$ for all our experimental cases can be found in Figure 18. From these data, it seems that there are no noticeable zones for which the compactness of the adhesive joint is lower. In other words, there is no part of the joint where pores are more abundant or bigger in the $(x y)$ plane. As such, this would imply that the shift in curing temperature does not induce any major changes in terms of planar distribution of the centroids of the created pores and that the concentration mechanisms which appear to exist are occurring along the thickness of the joint, as demonstrated by Figure 17. 


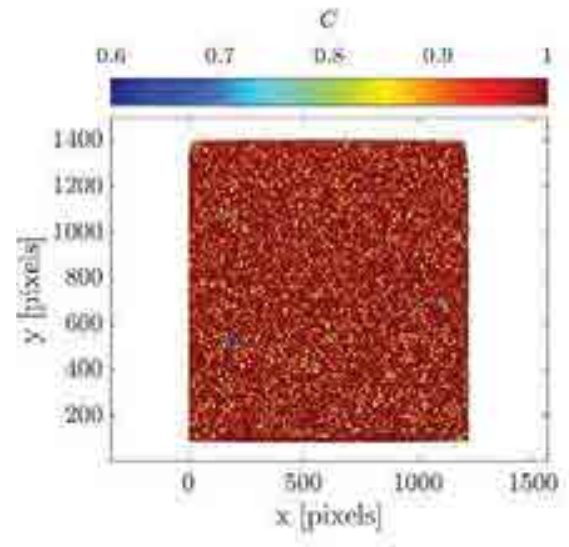

(a) Adhesive A, low temperature curing

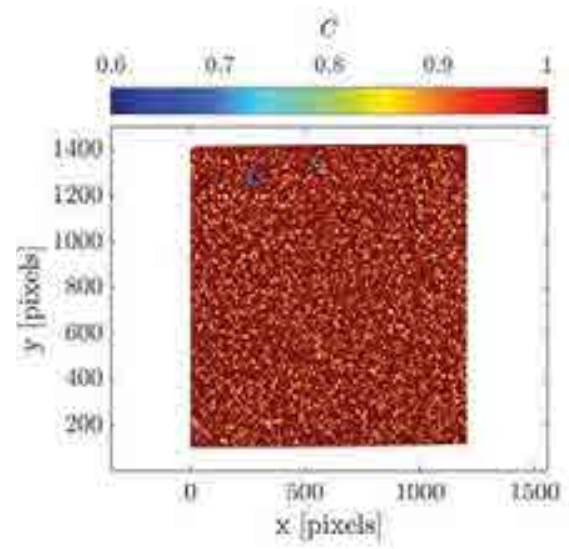

(c) Adhesive B, low temperature curing

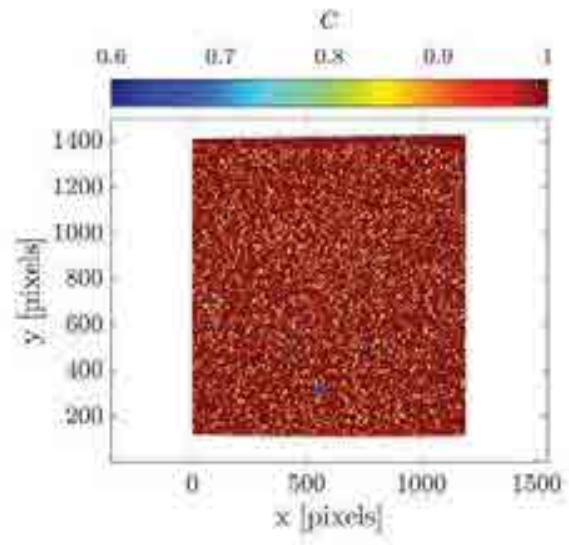

(b) Adhesive A, high temperature curing

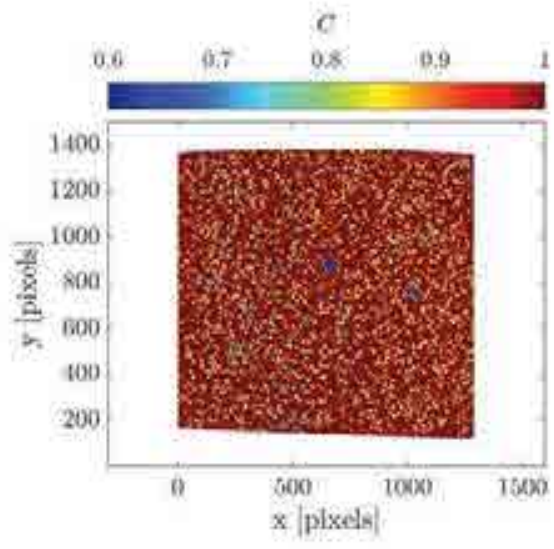

(d) Adhesive B, high temperature curing

Figure 18. Compactness maps computed for all the adhesive joints under investigation.

This can be confirmed by analysing the distributions of the coordinates of the pores for each segmented volume, as it is done in Figure 19. In these graphs, each one of the three plotted lines shows the distribution of the coordinates $x, y$ and $z$ respectively. The distribution of a given coordinate component ( $x$, for instance) is evaluated disregarding the values of the two others (i.e. $y$ and $z$ ). Moreover, these distributions are not evaluated in terms of numbers of individuals in each kernel, but in terms of volumetric fraction of each kernel with respect to the complete volume of pores. It should be noted that the count of each interval used to compute these distributions has been normalised with respect to the total number of individuals to obtain frequencies.

As it may be easily seen in Figure 19(a,d), there are no prevalent coordinates for the pores centroids along the $\vec{x}$ and $\vec{y}$ which corroborates the 


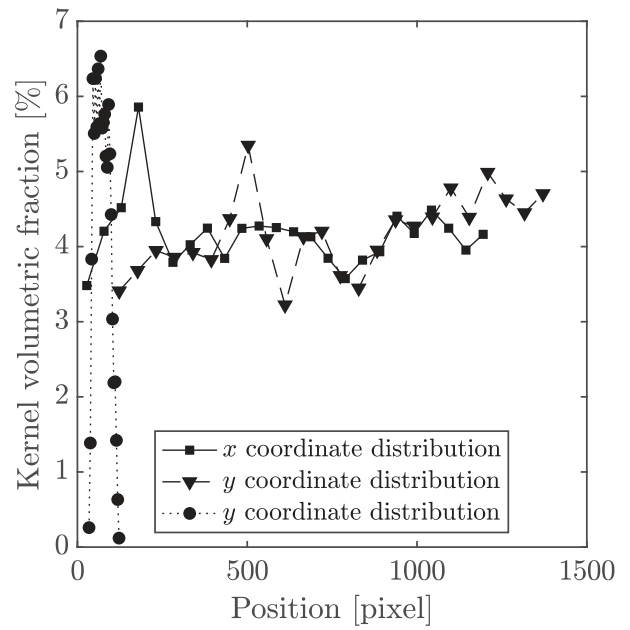

(a) Adhesive A, low temperature curing

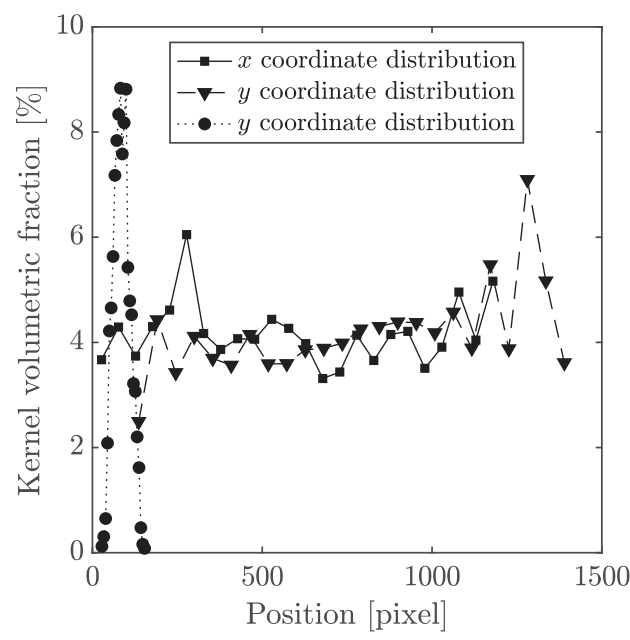

(c) Adhesive B, low temperature curing

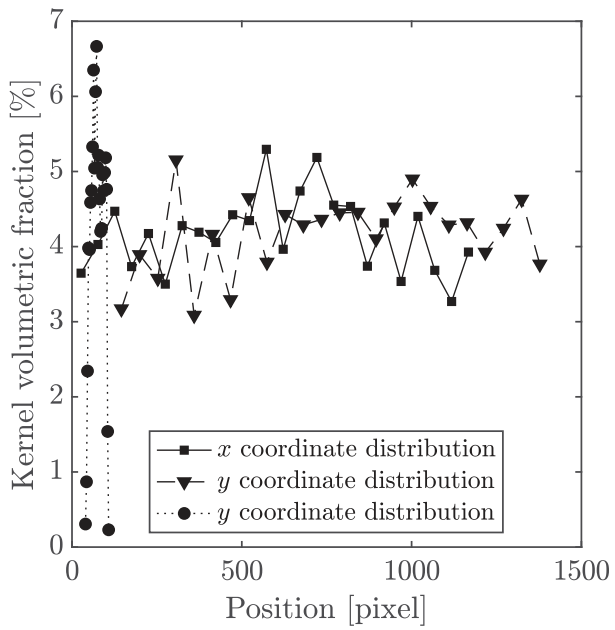

(b) Adhesive A, high temperature curing

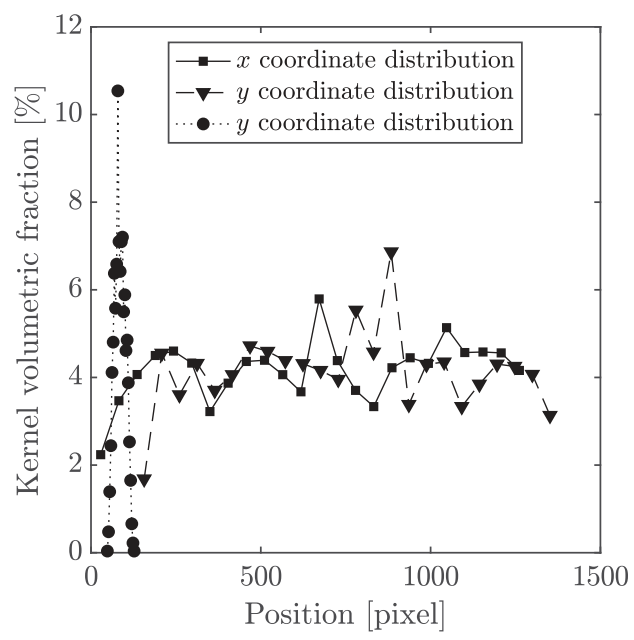

(d) Adhesive B, high temperature curing

Figure 19. Pores coordinates distributions within the adhesive joint.

observations done in Figure 18. In addition, the concentration of the pores in the middle of the joint following $\vec{z}$ can be quite clearly visualised, as expressed earlier in this paper (Figure 17).

\subsection{Local pores volumetric fraction}

The data displayed in Figure 19(a,d) provide with knowledge about the number of pores as a function of the coordinates, but any volumetric ratio consideration is left aside in this approach. In order to obtain such information, the computation of a local porosity ratio is suggested, hereafter referred to as $\eta_{l o c}$, in a sliding window sweeping the complete volume. The dimensions of said window can be selected 
arbitrarily, but it was chosen to use the sizes of the pores as a basis for their calculation. The window used to compute these local ratios needs to be sufficiently large to not be outweighed by large pores (i.e. not to yield cases where there are only pore-related pixels in the window), but at the same time it needs to be small enough to remain local. As such, a window size $\Delta$ defined with respect to the statistics computed on the diameters distributions is used for each studied case, as expressed in Eq.7. The resulting kernel sizes vary between 20 and 29 pixels. The size of the window along $\vec{z}$ is set equal to the joint thickness. Therefore, $\eta_{l o c}$ will be computed in $[\Delta, \Delta, p]$ subvolumes within the complete adhesive joint.

$$
\Delta=\left\lfloor\mu_{d}+4 \sigma_{d}\right\rfloor
$$

where $\mu_{d}$ and $\sigma_{d}$ are the average diameter and the diameters standard deviation for a given pores dataset.

The value maps for $\eta_{l o c}$ can be found in Figure 20. These maps are to be linked with the data presented in Figure 18(a,d), as they give similar but complementary information. Several conclusions may be extracted from these figures: (i) a higher curing temperature does not induce local increase of porosity ratio, (ii) the physico-chemical properties of the investigated adhesives do not impact this quantity for the studied cases, and (iii) the only concentration zones are linked to high diameters pores. These larger pores are hopefully the most uncommon as well (as demonstrated by the diameters distributions displayed in Figure 14) and usually created while spreading the adhesive on the bonding surfaces. There does not seem to be a general trend once these peculiar values put aside, leading the authors to conclude that there are no favoured sites for pores concentrations in the place of the joint, may it be in terms of number or in terms of volumetric fraction.

Very high values for $\eta_{l o c}$ are reached in all the studied cases (up to $75 \%$ for the adhesive $\mathrm{B}$ cured at $110^{\circ} \mathrm{C}$ ), comforting the hypothesis that pores within an adhesive joint are, to some extent, a threat to the mechanical strength of the bonded structure.

\section{Conclusion}

Adhesively bonded samples were made for two different curing cycles and two different bicomponent epoxy adhesives. These samples were placed in the chamber of an X-ray tomograph, in order to characterise the microstructure of the adhesive joints. The goal of these measurements was to detect the pores created during the bonding and during the curing inside the adhesive joints, and to investigate the potential change of their properties depending on the adhesive formulation and on the polymerisation cycle used to cure the materials. A segmentation-oriented image processing tool was then developed, so as to process the resulting CTscans. This tool has been tried on artificial datasets to ensure its performances and to check its limitations. There are multiple findings for this study. 


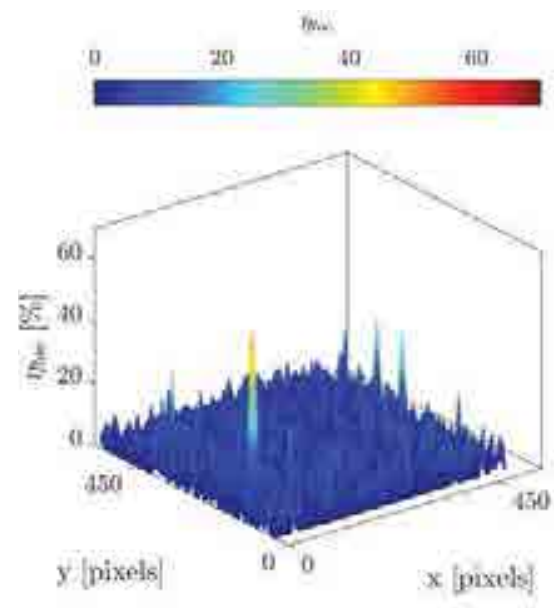

(a) Adhesive A, low temperature curing
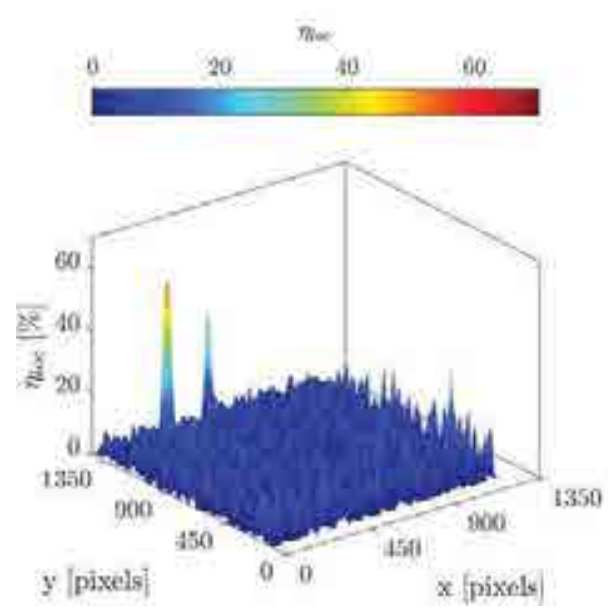

(c) Adhesive B, low temperature curing

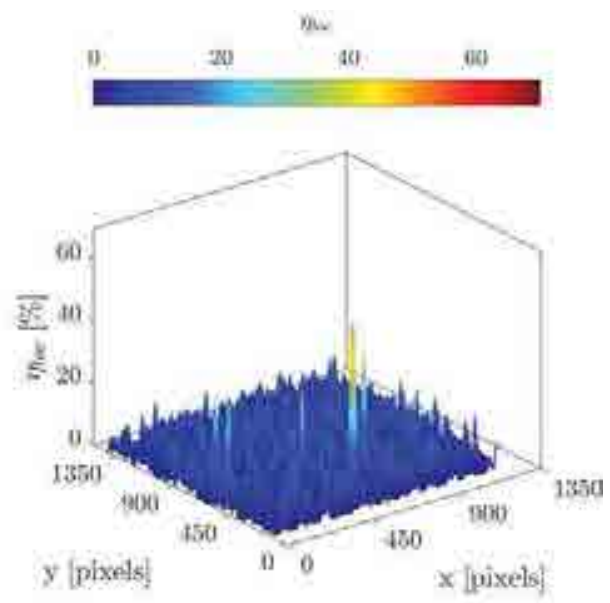

(b) Adhesive A, high temperature curing
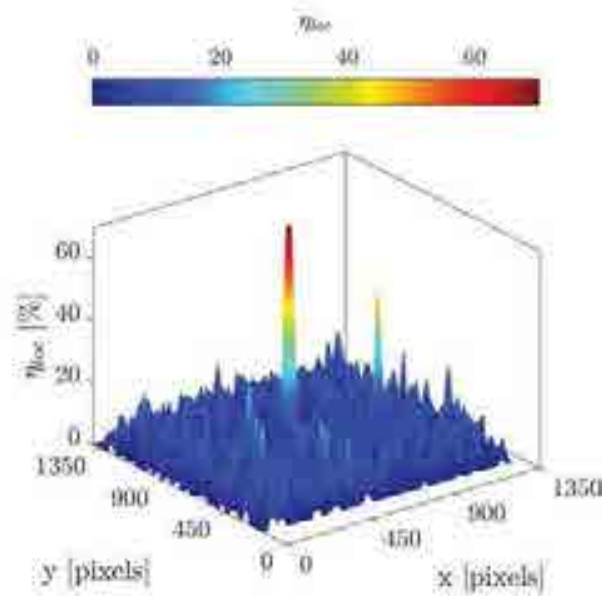

(d) Adhesive B, high temperature curing

Figure 20. Local pores volumetric fractions.

First, it has been shown that the curing cycle and the adhesive formulation could have significant influences on the pores created during the manufacturing of the joint. These differences could be explained by the disparities between the adhesives in terms of texture and chemical compositions, but no tangible answer can be provided at this stage of the study regarding these aspects. However, among the two adhesives tested, one seemed to be relatively insensitive to the curing conditions (adhesive A), as little to no difference was experienced for the curing cycles used in this study. This implies that different adhesives may have different sensitivities to the bonding and curing conditions, but the factors driving this sensitivity remain unknown so far. Further information could be obtained through in-situ X-ray microtomography observations during the curing of the materials. Particularly, such experiments would provide interesting data regarding the effect of the viscosity changes which happen during the curing, due to the increase in 
temperature and to the cross-linking reaction. Directly related to the curing reaction, the effects of the chemical shrinkage and of the residual stresses should also be investigated, in spite of their relative difficulty to be quantified.

It was also shown that pores are likely to be concentrated halfway through the thickness of the adhesive joint. Depending on the porosity volume fraction, this could be a factor to take into account in mechanical dimensioning computations, as the cross-section of the joint could be decreased by a noticeable and easily detectable amount, and the proximity of several large pores could also create preferred crack propagation paths since the adhesive bond would be locally weaker. Therefore, it would make sense, for mechanical strength reasons, to decrease as much as possible their creation during the bonding and the curing of the adhesives, especially for critical industrial fields such as aeronautics or space industry. Depending on the sensitivity of the material to the curing temperature, this decrease in pores number and volumetric ratio could be achieved by optimising the curing cycle so as to be as complete as possible in terms of polymerisation rate of the adhesive, and at the same time to be as low-temperature as possible. It is common for adhesives manufacturer to provide advised curing cycles in their datasheets, some of them being high-temperature cycles. This study shows that even if these advised cycles result in a fully polymerised material, the induced microstructure could trigger premature failure mechanisms, not necessarily for basic loadings such as monotonic or even creep, but perhaps for cyclic and fatigue loadings, or any long-term loading from a general standpoint. Working on the curing cycles is however not the only solution, as it is conceivable to mix the adhesive components in a vacuum environment, for example, which could decrease the pores created during the mixing.

\section{ORCID}

V. Dumont (10) http://orcid.org/0000-0001-6214-3451

\section{References}

[1] Moussa, O.; Vassilopoulos, A. P.; Keller, T. Effects of Low-temperature Curing on Physical Behavior of Cold-curing Epoxy Adhesives in Bridge Construction. Int. J. Adhes. Adhesives. 2012, 32, 15-22. DOI: 10.1016/j.ijadhadh.2011.09.001.

[2] Adams, R. D. Adhesive Bonding Science, Technology and Applications; Woodhead Publishing Ltd Elsevier, 2005.

[3] Da Silva, L. F. M. Modeling of Adhesively Bonded Joints; Springer-Verlag Berlin Heidelberg, 2008.

[4] Liu, X.; Bathias, C. Defects in Squeeze-cast Al2O3/Al Alloy Composites and Their Effects on Mechanical Properties. Compos. Sci. Technol. 1993, 46, 245-252. DOI: 10.1016/02663538(93)90158-D.

[5] Hirano, T.; Usami, K.; Tanaka, Y.; Masuda, C. In Situ X-ray CT under Tensile Loading Using Synchrotron Radiation. J. Mater. Res. 1995, 10, 381386. DOI: 10.1557/JMR.1995.0381. 
[6] Cottrino, S.; Jorand, Y.; Maire, E.; Adrien, J. Characterization by X-ray Tomography of Granulated Alumina Powder during in Situ Die Compaction. Mater. Charact. 2013, 81, 111-123. DOI: 10.1016/j.matchar.2013.04.004.

[7] Maire, E.; Carmona, V.; Courbon, J.; Ludwig, W. Fast X-ray Tomography and Acoustic Emission Study of Damage in Metals during Continuous Tensile Tests. Acta Mater. 2007, 55, 6806-6815. DOI: 10.1016/j.actamat.2007.08.043.

[8] Adrien, J.; Maire, E.; Gimenez, N.; Sauvant-Moynot, V. Experimental Study of the Compression Behaviour of Syntactic Foams by in Situ X-ray Tomography. Acta Mater. 2007, 55, 1667-1679. DOI: 10.1016/j.actamat.2006.10.027.

[9] Maire, E.; Fazekas, A.; Salvo, L.; Dendievel, R.; Youssef, S.; Cloetens, P.; Letang, J. M. X-ray Tomography Applied to the Characterization of Cellular Materials. Related Finite Element Modeling Problems. Compos. Sci. Technol. 2003, 63, 2431-2443. DOI: 10.1016/ S0266-3538(03)00276-8.

[10] Pavan, M.; Craeghs, T.; Verhelst, R.; Ducatteeuw, O.; Kruth, J. P.; Dewulf, W. CT-based Quality Control of Laser Sintering of Polymers. Case Stud. Non-Destruct. Test. Eval. 2016, 6, 62-68. DOI: 10.1016/j.csndt.2016.04.004.

[11] Wang, X.; Zhao, L.; Fuh, J. Y. H.; Lee, H. P. Effect of Porosity on Mechanical Properties of 3D Printed Polymers: Experiments and Micromechanical Modeling Based on X-ray Computed Tomography Analysis. Polymers. 2019, 11. DOI: 10.3390/polym11071154.

[12] McKinley, P.; Ching, D.; Kamke, F.; Zauner, M.; Xiao, X. Micro X-ray Computed Tomography of Adhesive Bonds in Wood. Wood Fiber Sci. 2016, 48, 2-16.

[13] Schwarzkopf, M.; Muszynski, L. Strain Distribution and Load Transfer in the Polymer-wood Particle Bond in Wood Plastic Composites. Holzforschung. 2015, 69, 53-60. DOI: 10.1515/hf-2013-0243.

[14] Taud, H.; Martinez-Angeles, R.; Parrot, J. F.; Hernandez-Escobedo, L. Porosity Estimation Method by X-ray Computed Tomography. J. Petrol. Sci. Eng. 2005, 47, 209-217. DOI: 10.1016/j.petrol.2005.03.009.

[15] Pinzer, B. R.; Medebach, A.; Limbach, H. J.; Dubois, C.; Stampanoni, M.; Schneebeli, M. 3D-characterization of Three-phase Systems Using X-ray Tomography: Tracking the Microstructural Evolution in Ice Cream. Soft Matter. 2012, 8, 4584. DOI: 10.1039/ c2sm00034b.

[16] Little, J. E.; Yuan, X.; Jones, M. I. Characterisation of Voids in Fibre Reinforced Composite Materials. NDT E Int. 2011, 46, 122-127. DOI: 10.1016/j.ndteint.2011.11.011.

[17] Alonso-Sierra, S.; Velázquez-Castillo, R.; Millán-Malo, B.; Nava, R.; Bucio, L.; ManzanoRamírez, A.; Cid-Luna, H.; Rivera-Muñoz, E. M. Interconnected Porosity Analysis by 3D $\mathrm{X}$-Ray Microtomography and Mechanical Behavior of Biomimetic Organic-Inorganic Composite Materials. Mater. Sci. Eng. C. 2017. DOI: 10.1016/j.msec.2017.05.106.

[18] Plank, B.; Mayr, G.; Reh, A.; Kiefel, D.; Stoessel, R.; Kastner, J. Evaluation and Visualisation of Shape Factors in Dependence of the Void Content within CFRP by Means of X-ray Computed Tomography. tech. rep.; 2014.

[19] Enns, J. B.; Gillham, J. K. Time-Temperature-Transformation (TTT) Cure Diagram: Modeling the Cure Behavior of Thermosets. J. Appl. Polym. Sci. 1983, 28, 2567-2591. DOI: 10.1002/app.1983.070280810.

[20] Buffiere, J.-Y.; Maire, E.; Adrien, J.; Masse, J.-P.; Boller, E. In Situ Experiments with X Ray Tomography: An Attractive Tool for Experimental Mechanics. Exp. Mech. 2010, 50, 289-305. DOI: 10.1007/s11340-010-9333-7.

[21] Kak, A. C.; Slaney, M. Principles of Computerized Tomographic Imaging; Society for Industrial and Applied Mathematics: Philadelphia, 2001. 
[22] Dumont, V.; Badulescu, C.; Adrien, J.; Carrere, N.; Thévenet, D.; Maire, E. Experimental Investigation of Porosities Evolution in a Bonded Assembly by Means of X-Ray Tomography. J. Adhes. 2019. DOI: 10.1080/00218464.2019.1685984.

[23] Sezgin, M. Survey over Image Thresholding Techniques and Quantitative Performance Evaluation. J. Electron. Imaging. 2004, 13, 146-165. DOI: 10.1117/1.1631315.

[24] Rosenfeld, A.; De La Torre, P. Histogram Concavity Analysis as an Aid in Threshold Selection. IEEE Trans. Syst. Man Cybern. 1983, 13, 231-235. DOI: 10.1109/ TSMC.1983.6313118.

[25] Otsu, N.;. A Threshold Selection Method from Gray-Level Histograms. IEEE Trans. Syst. Man Cybern. 1979, 9, 62-66. DOI: 10.1109/TSMC.1979.4310076.

[26] Lim, J. S. Two-dimensional Signal and Image Processing; Hall, P. Ed., 1990; pp. 469-476. Upper Saddle River, NJ: Prentice-Hall, Inc.

[27] Gallagher, N. C.; Wise, G. L. A Theoretical Analysis of the Properties of Median Filters. IEEE Trans. Acoust. Speech Signal Process. 1981, 29, 1136-1141. DOI: 10.1109/ TASSP.1981.1163708.

[28] Schindelin, J.; Arganda-Carreras, I.; Frise, E.; Kaynig, V.; Longair, M.; Pietzsch, T.; Preibisch, S.; Rueden, C.; Saalfeld, S.; Schmid, B. Fiji: An Open-source Platform for Biological-image Analysis. Nat. Methods. 2012, 9, 676 EP. DOI: 10.1038/nmeth.2019.

[29] Wadell, H. Volume, Shape, and Roundness of Quartz Particles. J. Geol. 1935, 43, 250-280. DOI: $10.1086 / 624298$. 